\title{
THE IMPORTANCE OF LOCALLY CONVERTED WAVES ON THE ELASTIC INVERSION OF COMPRESSIONAL DATA
}

\author{
Jorge Nicolás Hounie ${ }^{1}$ and Sérgio Adriano Moura Oliveira²
}

\begin{abstract}
Seismic inversion is routinely used in oil and gas exploration to estimate the elastic properties of the subsurface. However, most of the inversion methods used in the industry disregard an inherent phenomenon of wave propagation in elastic media: the conversion of compressional waves into shear waves and vice versa. In this paper we analyze the importance of the locally converted seismic waves in the results of compressional wave based elastic inversion. For this, the reflectivity method is used to model the seismic response of a layered elastic media and also as the base of a nonlinear inversion method. We show that the compressional waves generated by local conversion can hardly be identified and eliminated by moveout filters once their transit time are very close to that of primary reflections. To assess the impact of the locally converted waves, two versions of the inversion method were implemented: in the first one, all seismic events generated in a stratified medium were taken into account, including the effects of transmission, internal multiples and converted waves. In the second version, the converted waves were ignored. A series of synthetic data were generated using full reflectivity modeling and submitted to the two versions of the inversion methods, what allow us to evaluate the error made when these waves are ignored. We conclude that this error is proportional to the degree of contrast in elastic properties between layers and is greatly affected by the presence of thin layers.
\end{abstract}

Keywords: waveform inversion, reflectivity, seismic.

RESUMO. A inversão de dados sísmicos é utilizada rotineiramente na exploração de hidrocarbonetos e na caracterização de reservatórios, com o objetivo de estimar as propriedades elásticas do interior da terra. No entanto, a maioria dos algoritmos de inversão elástica desconsidera um fenômeno inerente à propagação de ondas em meios elásticos: a conversão de ondas compressionais em cisalhantes e vice-versa. Neste artigo analisamos o impacto das ondas convertidas localmente nos resultados de inversão elástica de dados compressionais. 0 método da refletividade é utilizado de duas maneiras na análise das ondas convertidas: como método de modelagem e como base para algoritmos de inversão. A modelagem mostra que as ondas convertidas localmente são de difícil identificação em um sismograma, confundindo-se com eventos de origem puramente compressional, o que torna inviável sua filtragem com os métodos de processamento baseados na diferença de tempo de trânsito entre eventos compressionais e convertidos. Para avaliar a influência das ondas convertidas nos resultados da inversão elástica de dados compressionais foram desenvolvidas duas estratégias de inversão baseadas no método da refletividade: na primeira, foram considerados todos os efeitos de propagação em meios elásticos estratificados, enquanto na segunda foram desconsideradas as conversões de modo que ocorrem entre as camadas. As estratégias foram testadas em dados sintéticos, e os resultados permitiram avaliar a influência das ondas convertidas na inversão elástica de dados compressionais. De uma maneira geral, a inversão de forma de onda considerando todos os efeitos de propagação apresentou resultados superiores aos da inversão que desconsideram as conversões de modo. Entretanto, em situações onde não ocorram camadas delgadas com grandes contrastes nas propriedades em relação às camadas adjacentes, as duas estratégias apresentaram resultados similares.

Palavras-chave: inversão de forma de onda, refletividade, sísmica.

\footnotetext{
1 Petrobras, UO-BC/EXP/GEOF, Av. Elias Agostinho, 665, CPD $1{ }^{\circ}$ andar, Imbetiba, Macaé, RJ, Brazil. Phone: +55(22) 2761-6529 - E-mail: jnicolashe@gmail.com

2Laboratório de Engenharia de Exploração e Produção de Petróleo, Universidade Estadual do Norte Fluminense (LENEP/UENF) e Instituto Nacional de Ciência e Tecnologia em Geofísica do Petróleo (INCT-GP/CNPq), Av. Brennand, s/n, Imboassica, 27925-310 Macaé, RJ, Brazil. Phone: +55(22) 2765-6567; Fax: +55(22) 2765-6551

- E-mail: moura@lenep.uenf.br
} 


\section{INTRODUCTION}

Seismic inversion is a technique that allows estimating the elastic properties of rocks from seismic data. In recent years this technique has played an increasing role in hydrocarbon exploration and seismic reservoir monitoring. The results obtained by the inversion can be interpreted in terms of interval properties, which facilitate the integration of seismic and geological information. In addition, the elastic properties estimated in the inversion combined with well logs and petrophysical relationships allow us to estimate many fundamental parameters for reservoir characterization, such as porosity and saturation of the rocks.

Currently the most widely used approach for estimating the elastic parameters from seismic data is AVO (amplitude versus offset) inversion. This technique is based on an approximation for the plane wave reflection coefficients between two elastic media derived from the Zoeppritz equations. The main limitation of this method is that it considers that the seismic signal is formed by primary reflections only, not taking into account transmission effects, mode conversion and internal multiples, which depending on the case, may be very important (Simmons \& Backus, 1994; Mallick, 2007).

In this article we analyze the impact of locally converted waves in the elastic inversion results. Our strategy to achieve this goal was to develop a method of model based waveform inversion that estimates the elastic parameters of the layers minimizing the difference between the observed and modeled data. This method is not affected by the simplifications assumed in AVO inversion and the angle range of the input data doesn't need to be restricted to the values for which the approximations commonly used for the reflection coefficient are valid, smaller than the critical angle. Also, in our implementation, we can take or not into account the converted waves. We apply it on synthetic data containing all events generated in a layered elastic media, and in the inversion we evaluated the impact of considering or not the converted waves in the calculations of the Jacobian matrix and gradient vector.

The modeling and inversion methods developed in this paper are based on the reflectivity method, which is used to model the plane wave response of an elastic layered media. One interesting characteristic of this method is that it can be used to model the complete response of the media (including primaries, multiples and conversions), but it also can be modified in order to model the partial response and not take into account the converted waves, whose influence on amplitudes and in the estimation of elastic parameters will be analyzed in this work. The elastic inversion method developed in this work uses the Levenberg Marquadt algorithm in order to minimize the objective function. To this we developed an analytical procedure to calculate the partial derivatives of the data with respect to the elastic parameters. These derivatives are calculated concurrently with the reflectivity of the medium, which increased the computational efficiency of the inversion algorithm.

The inversion and modeling procedures were designed to deal with compressional waves resulting from marine seismic acquisition where both the source and receivers are located in the water layer, and the receivers register the pressure variations, so we treat converted compressional waves resulting from conversion of the descendent $P$ wave into $S$ wave in the layers that converts again to ascendant $P$ waves.

\section{ELASTIC INVERSION BASED ON THE COMPLETE AND PARTIAL REFLECTIVITY METHOD}

In the examples shown in this work we consider the earth as a 1-D layered media. The objective of the elastic seismic inversion is to determine the $P$ wave velocity, $S$ wave velocity and density of each layer. The inversion method developed in this work is similar to the ones developed by Zhao et al. (1994) and Gouveia (1996). However, we present a new analytical method to calculate the Jacobian matrix (see Appendix B). The reflectivity method is a mathematical method that is used to model the propagation of elastic waves in 1-D layered media. This is a recursive method that models the full response of the media, and its recurrence formula may be simplified in order to not take into account some specific events (see Oliveira, 2009). This method is the heart of our model based inversion algorithm which can take into account or not the locally converted waves, so we can check the impact of this event into the final inversion result.

Our elastic waveform inversion is a nonlinear method that estimates the elastic parameters by the minimization of an objective function that measures the quadratic error between the observed and calculated data. This objective function is given by the following formula:

$$
E(m)=\frac{1}{2} \Delta D^{T} \Delta D^{*}
$$

where $m$ is the parameter vector and $\Delta D$ is a column vector whose elements are the differences between the calculated and observed data samples. The inversion method operates in the plane wave $(\omega-p)$ domain so the data belongs to complex domain and $\Delta D^{*}$ represents the conjugate of $\Delta D$. In order to bring the real data to the $\omega-p$ domain it is necessary to apply a $\tau-p$ transform on it followed by a Fourier transform.

The Jacobian matrix contains the derivatives of the data with respect to the model parameters. We developed an analytical 

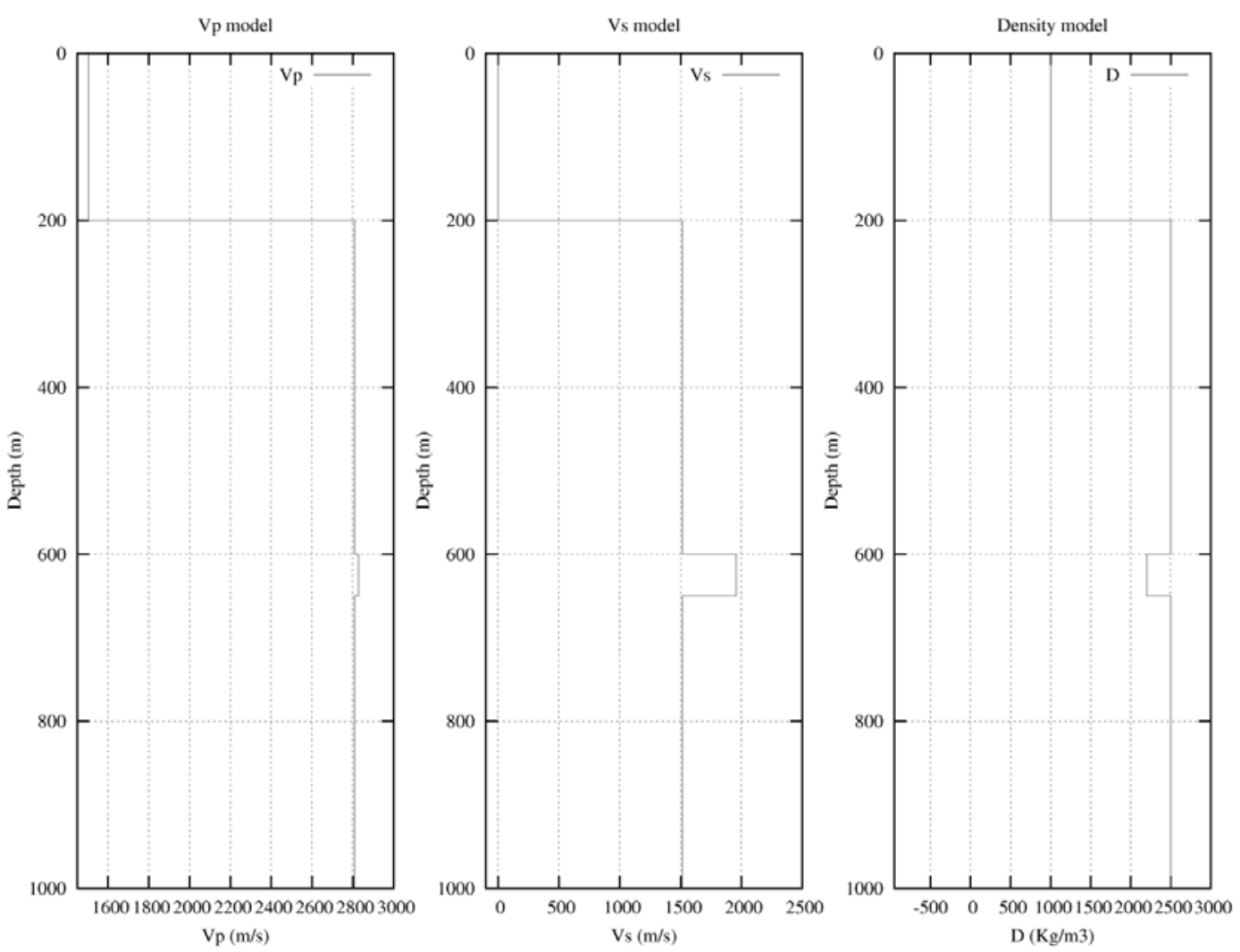

Figure 1 - Four-layer model: a water layer and a sedimentary section composed of a $50 \mathrm{~m}$ sandstone encased in shales.

procedure (Appendix A and B) to calculate these derivatives using the reflectivity method for the cases where the converted waves are taken into account and not. The minimization of the objective function is based on the Levenberg Marquadt algorithm, that is an iterative procedure where the following linear system needs to be solved in each iteration:

$$
\left[\Re\left(\mathbf{J}^{\mathbf{T}} \mathbf{J}^{*}\right)+\lambda \mathbf{I}\right] \delta m_{k}=-\Re\left(\mathbf{J}^{\mathbf{T}} \Delta D^{*}\right)
$$

after which the parameter vector is updated: $m_{k+1}=m_{k}+$ $\delta m_{k}$. The method needs an initial solution that contains the low frequency trend of the $V p, V s$ and density model. More details about the method can be found in Hounie (2011).

\section{RESULTS}

In this section we evaluate the impact of converted waves in the elastic inversion results by means of tests with synthetic data. First we apply the method in a simple four-layer model, in which we vary the thickness of the layers and the contrast in elastic properties to verify the influence of these parameters when we take or not into account the converted waves. After this we repeat the tests on synthetic data obtained from a more elaborate model that is representative, in terms of the elastic properties, of siliciclastic sediments. For this test, we also analyze the inversion results obtained by the AVO method, where only primary reflections are considered.

The first model we use is formed by a water layer $200 \mathrm{~m}$ thick followed by an $800 \mathrm{~m}$ shale layer, in which a gas saturated sand with a thickness of $50 \mathrm{~m}$ is encased (Fig. 1). This model was also used to assess the difference between the total and partial reflectivity response, shown in Figure 2. Note that the seismograms are in $\tau-p$ domain. They were modeled with the reflectivity method including all events, except the free surface multiples. The ray parameters used corresponds to angles between 1 and 89 degrees. A Ricker pulse with $30 \mathrm{~Hz}$ dominant frequency was used as wavelet. The first event corresponds to the water bottom reflection, followed by the reflections at the top and bottom of the gas sand layer. The first two events are identical in the total and partial responses, as evidenced on the difference section. The converted waves contribution to the base of gas sand reflection can be noted by amplitude variations between the complete and partial responses, clearly identified in the difference section (Fig. 2). The other events that involve converted waves are easily distinguished by their larger travel times. This example demonstrates 

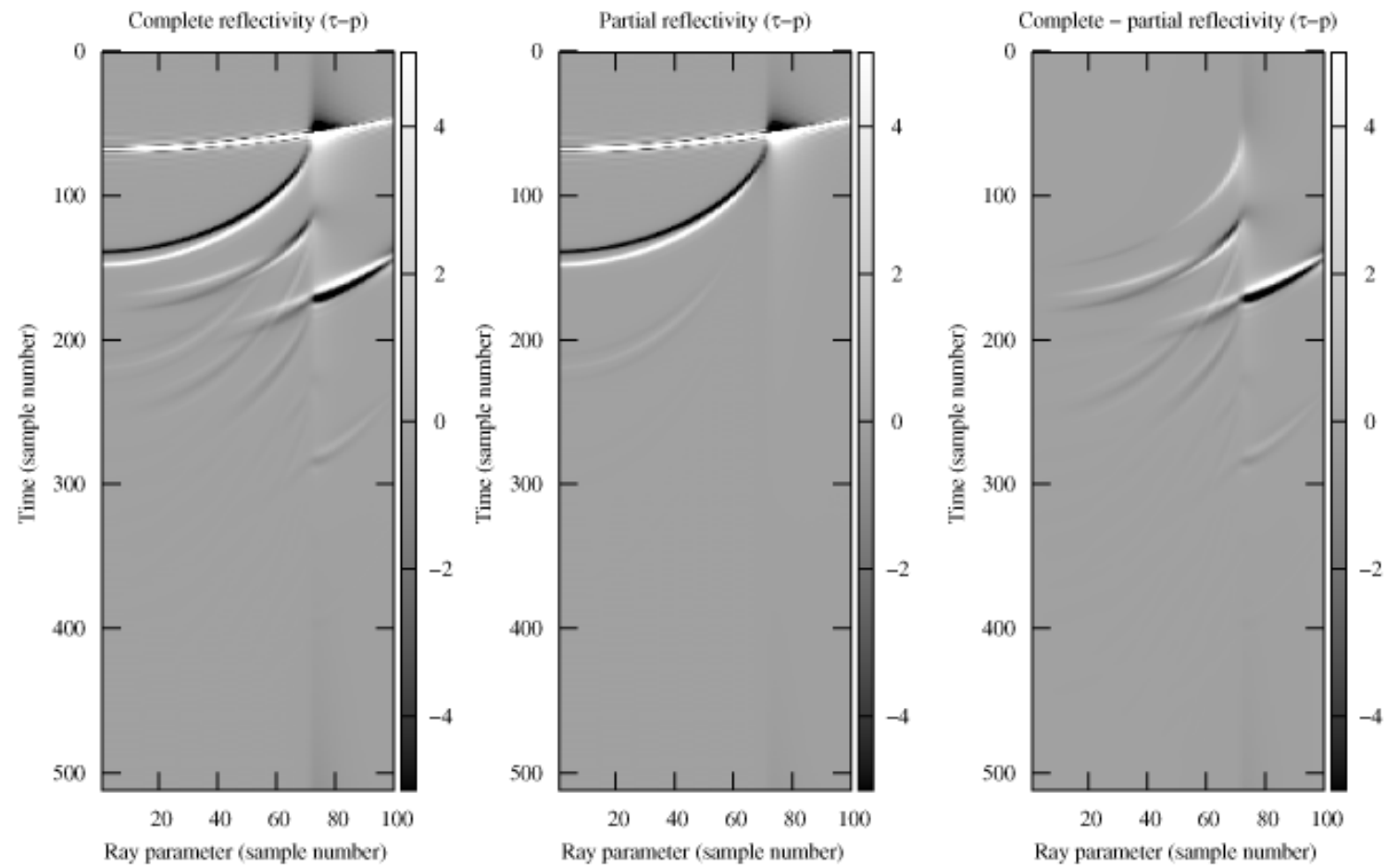

Figure 2 - Complete and partial $\tau-p$ responses to the model containing the $50 \mathrm{~m}$ sandstone layer in Figure 1 , and the difference between the two. The ray parameter range correspond to angles of emergence between 1 and 45 degrees.

that the converted waves can significantly change the amplitudes of events, as has been observed by Simmons \& Backus, 1994; Mallick, 2007.

The converted waves that propagate only in thin layers have very similar travel times to that of primary reflections and are known as locally converted waves. Such events can't be filtered by moveout filters due to insufficient travel time differences between them and primary events. This can be seen in Figure 3 that schematically shows many events generated in a thin layer.

Travel times were calculated in the $\tau-p$ domain for the depicted events in Figure 3, for incidence angles between 0 and 33 degrees. The model in Figure 1 was used. Travel times of primary reflections and locally converted waves in the sandstone layer are virtually indistinguishable (Fig. 4), forming a compound event. The travel time of the converted waves traveling in the thick shale layer above the sandstone allows them to be distinguished from the others events.

For inversion tests, the data were modeled from three different configurations of the four-layer model presented earlier. In case 1 the thickness of the sandstone layer was maintained at $50 \mathrm{~m}$. In case 2 it has been reduced to $25 \mathrm{~m}$ and in case 3 , the sandstone layer thickness was maintained at $25 \mathrm{~m}$ but the contrast in elastic properties between the sandstone and shale was reduced by half. The inversion was done in $\omega-p$ domain using frequencies components from 2 to $90 \mathrm{~Hz}$ and ray parameters that corresponded to angles from 1 to 45 degrees. For all cases, two types of results were obtained. One that accounts the locally converted waves in the inversion and other that does not. The elastic parameters estimated for each layer were $V p, V s$ and density. The nonlinear inversion method needed 16 iterations in order to attain convergence.

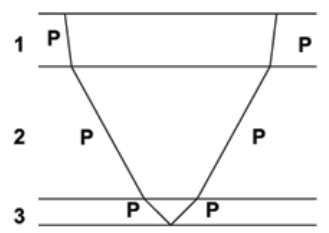

a)

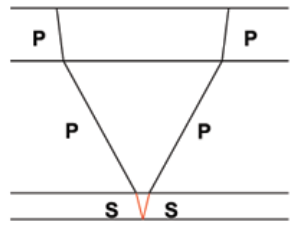

c)

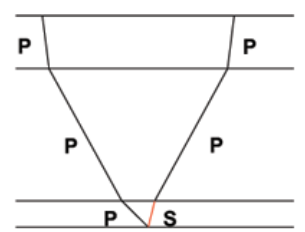

b)

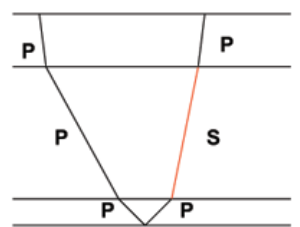

d)
Figure 3 - Seismic events propagating through a thin layer: a) only primary $P$ waves; b) $P S$ wave originated at the base of thin layer; c) $P S$ wave originated at top of thin layer and $S S$ reflection at the base; d) $P$ waves through thin layer and $P S$ wave propagating above thin layer. 


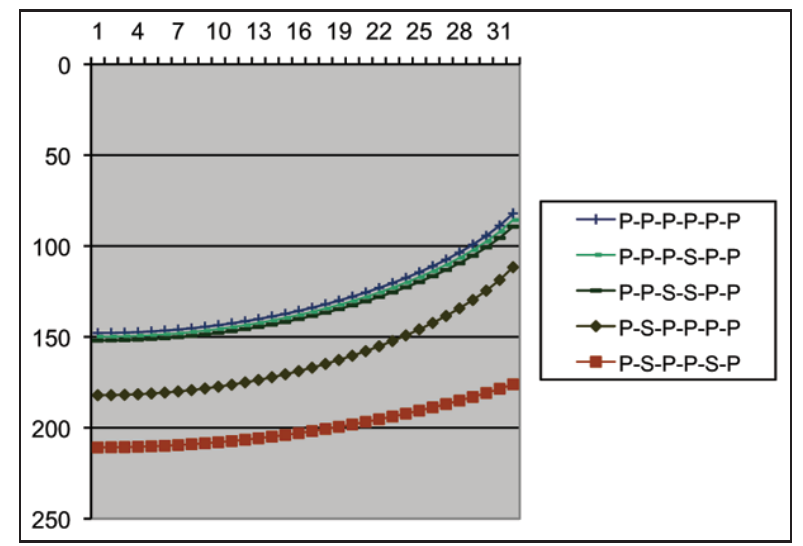

Figure 4 - Seismic events reflecting at the bottom of sandstone in Figure 1 in the $\tau-p$ domain. The letter sequence indicates the raypath. Events corresponding to cases a), b) and c) on Figure 3 have similar travel times.

\section{Results for case 1}

The inversion results obtained taking into account the converted waves are shown in Figures 5 and 6 . Very good results were observed for $V p, V s$ and density, since the values obtained by the inversion are very close to the target model. The results obtained by the inversion algorithm that does not take into account local conversions are shown in Figure 7. Notice that the recovered values of $V p, V s$ and density in the sandstone layer are close to the real one but some fluctuations have appeared below the sandstone layer. Analyzing the calculated and observed data (Fig. 8) we conclude that these oscillations are produced by false contrasts created by the inversion algorithm in order to try to adjust the events due to converted waves that occur below the primary reflections from the top and the base of the sandstone layer.

\section{Results for case 2}

The results of the complete response inversion for the test data generated from the $25 \mathrm{~m}$ sandstone layer can be seen in Figure 9. The inverted $V p, V s$ and density are not so close to the target model as in case 1. It also can be noticed that the inversion produced oscillations under and above the sandstone layer. The calculated and observed data are not as well adjusted as in case 1 (Fig. 10). However, the inversion that did not take into account the converted waves produced more oscillations in the inverted $V s$ and density models (Fig. 11). The density model obtained by the inversion that considered the converted waves is clearly superior. The simplified inversion for its turn creates oscillations in the models in order to generate $P P$ reflections to try to adjust events that are actually locally converted waves (Fig. 12).

\section{Results for case 3}

In case 3 the contrast in elastic properties between the sandstone and shale was reduced by half and, differently from last case, we note more similarities between the $V p, V s$ and density models produced by the inversion that take into account the locally converted waves and by the simplified inversion method (Figs. 13 and 15). The reduction in the elastic parameters contrast between the thin sandstone layer and the shale reduced the amplitudes of the locally converted waves compared with the amplitudes of primary $P P$ reflections (Figs. 14 and 16). This fact explains the similarity of the results produced by the two inversions.

\section{Model of a siliciclastic sedimentary section with moderate contrasts}

This model represents a portion of a typical siliciclastic sedimentary sequence, where the $V p / V s$ ratio is approximately constant and the relationship between $V p$ and density roughly follows the Gardner equation for shales. The thickness of each layer was kept constant at $10 \mathrm{~m}$. The $V p$ model was generated by adding random perturbations to a model with a constant speed of $2000 \mathrm{~m} / \mathrm{s}$. The $V s$ model was obtained from $V p$ considering a constant $V p / V s$ ratio and adding a random perturbation to this result. A similar procedure was used to create the density model, obtained from the Gardner equation for shales and then adding a random perturbation. The $V p, V s$ and density models are shown in Figure 17. The relationships between $V p$ and $V s$ and $V p$ and density are shown in Figure 18.

This model was used to generate a $P P$ synthetic seismogram of the complete response of the medium using the reflectivity method, with frequencies between 0 and $125 \mathrm{~Hz}$ and 200 ray parameters representing emergence angles between 1 and 89 degrees at the free surface. The free surface multiple were not modeled. A Ricker pulse with $30 \mathrm{~Hz}$ dominant frequency was used as wavelet.

The data used in the inversion were limited to an angle of incidence of 45 degrees. Constant values for $V p, V s$ and density representing average values of the model were used as the initial model. The number of iterations was limited to 16 and the pulse used in the inversion was identical to that used in the modeling.

The results obtained by the complete and partial response inversions were virtually identical, and both very close to the correct values, especially for $V p$ and $V s$. The density obtained from the inversion does not have the same quality as the results for $V p$ and $V s$, but still was able to recover most of the features of the original density model, and on several places the correct values 

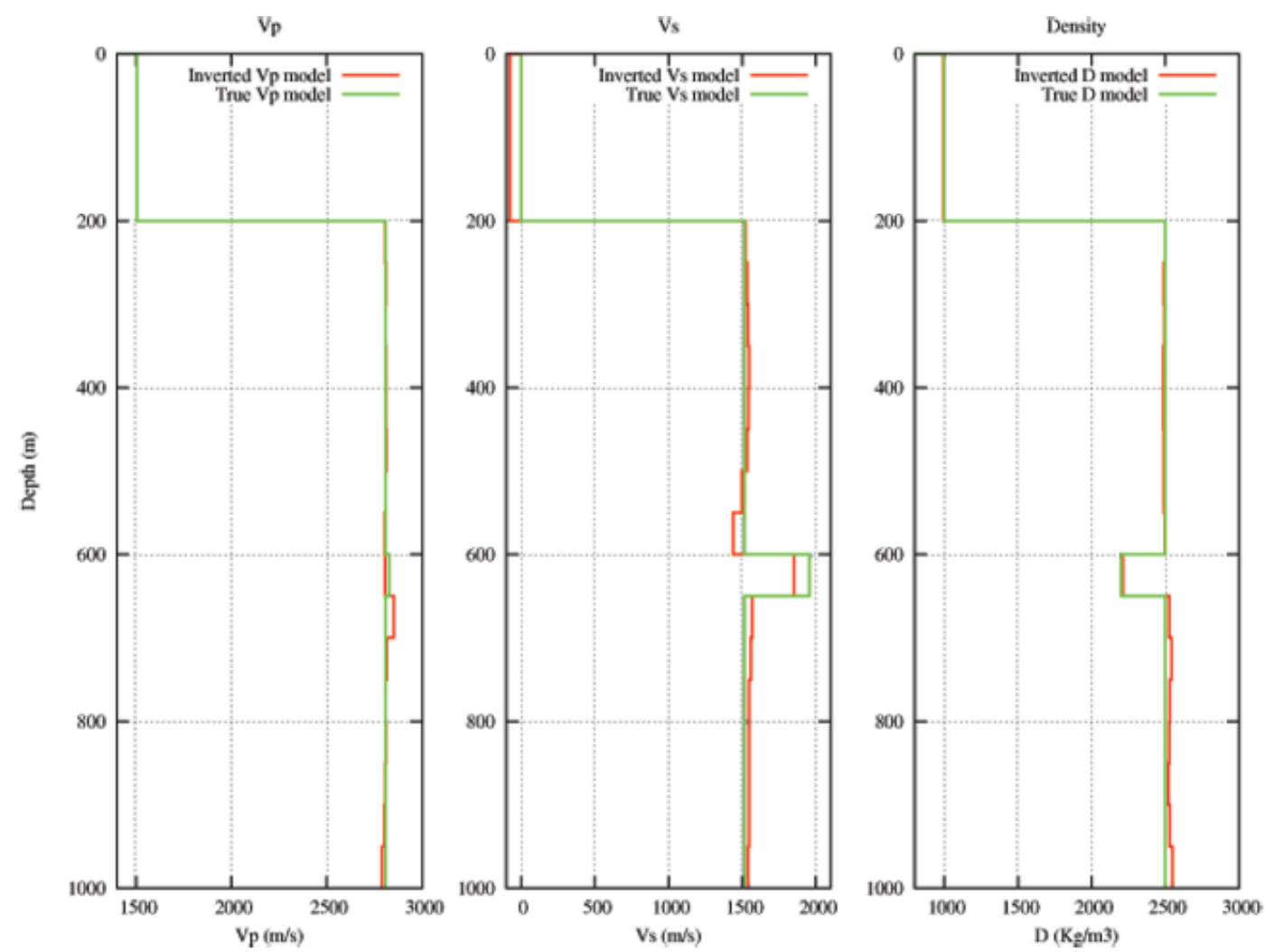

Figure $\mathbf{5}$ - Complete response inversion results for the $50 \mathrm{~m}$ sandstone model.

True model data

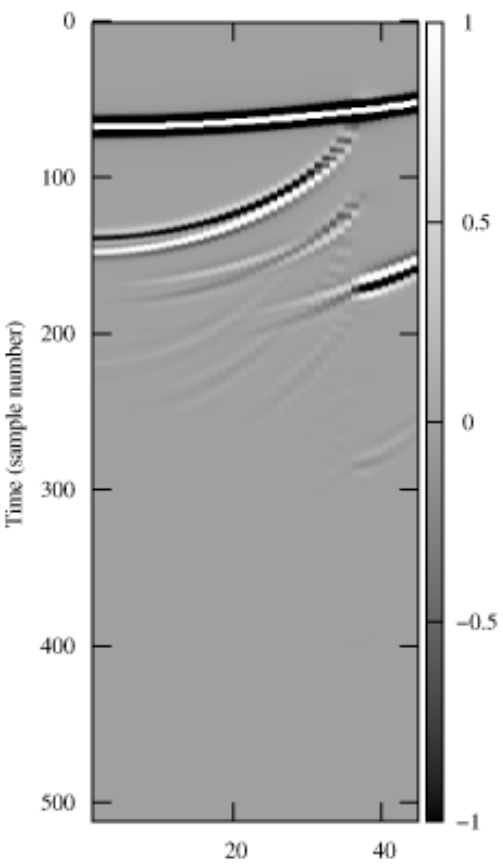

Ray parameter (sample number)
Inverted model data

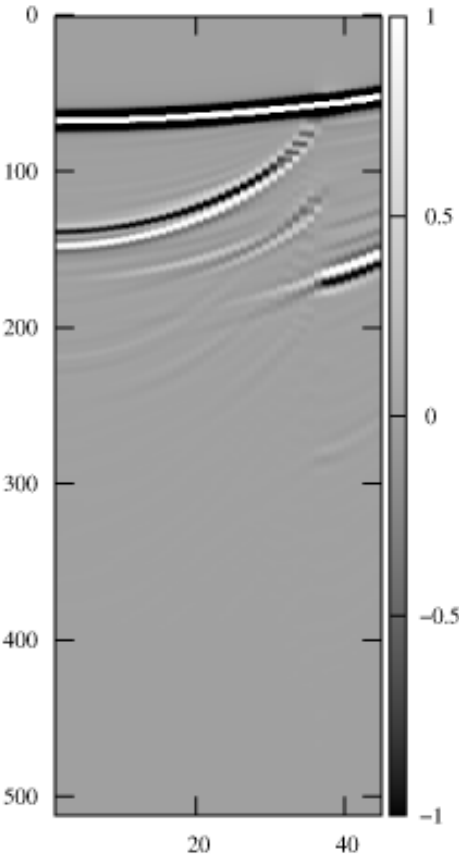

Ray parameter (sample number)

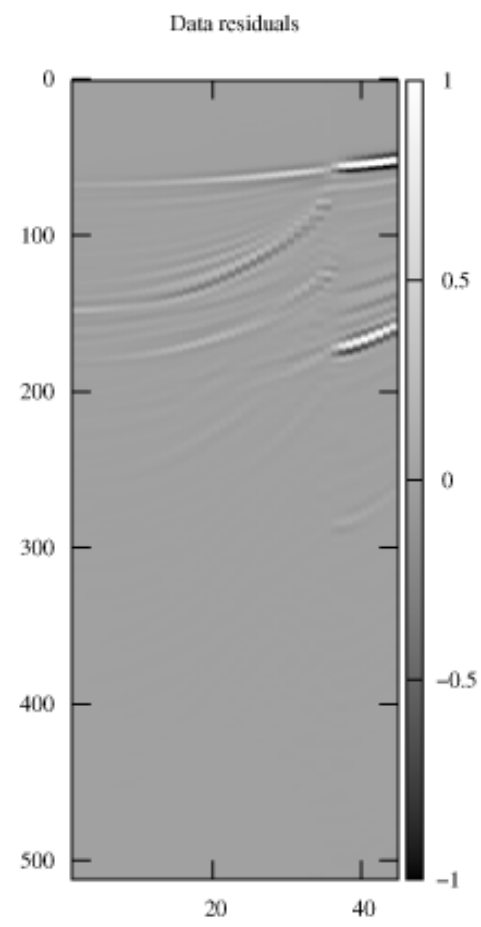

Ray parameter (sample number)

Figure 6 - Reference data; data from complete response inversion model; and data residuals for the $50 \mathrm{~m}$ sandstone model. 

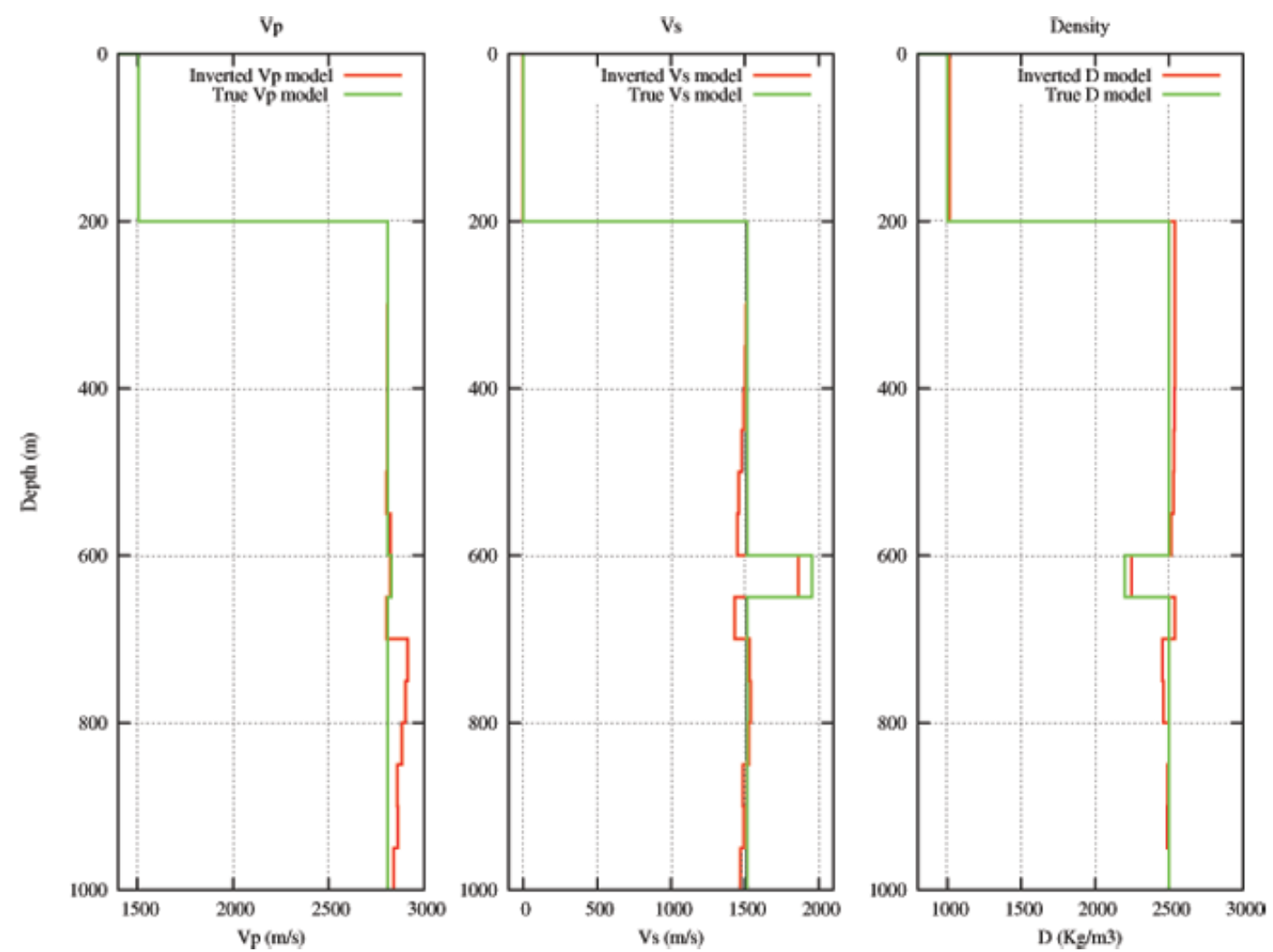

Figure 7 - Partial response inversion results for the $50 \mathrm{~m}$ sandstone model.

True model data

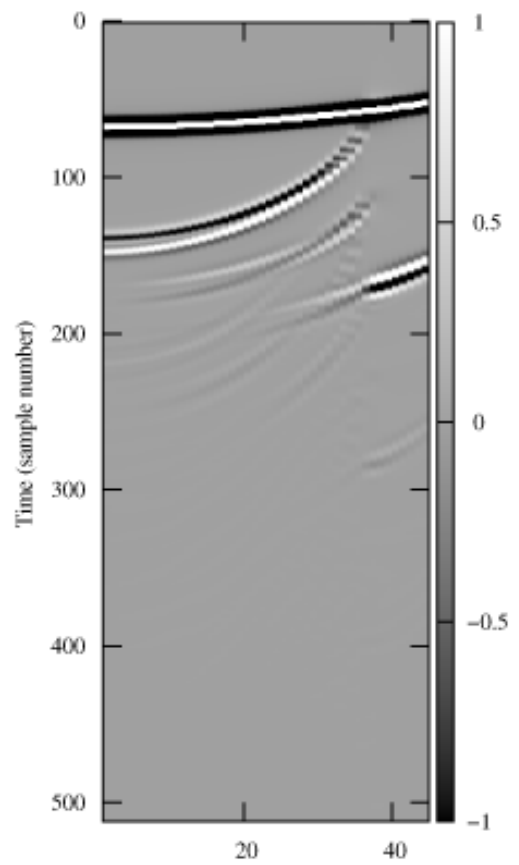

Ray parameter (sample number)
Inverted model data

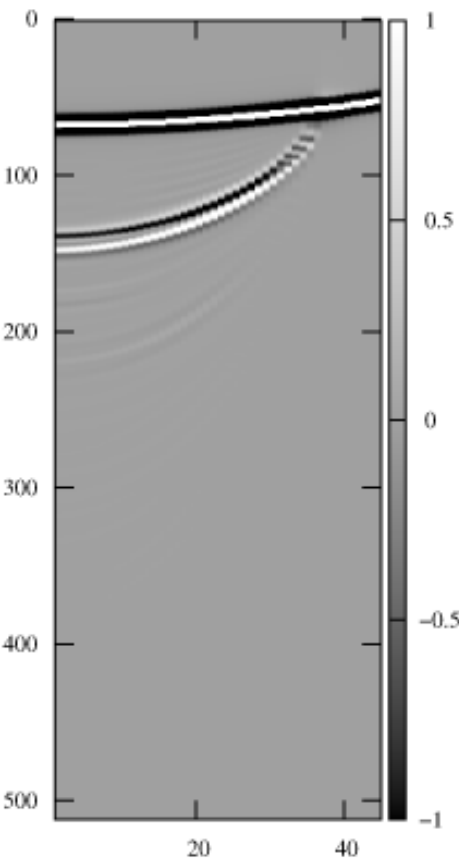

Ray parameter (sample number)
Data residuals

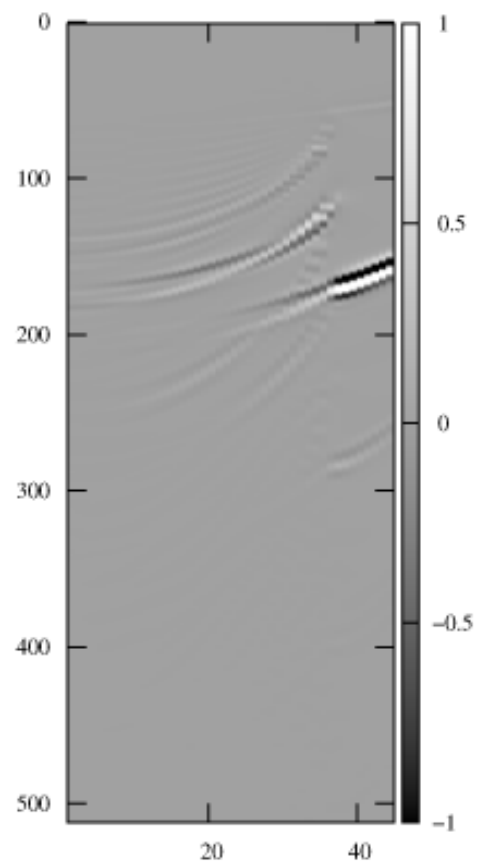

Ray parameter (sample number)

Figure 8 - Reference data; data from partial response inversion model; and data residuals for the $50 \mathrm{~m}$ sandstone model. 

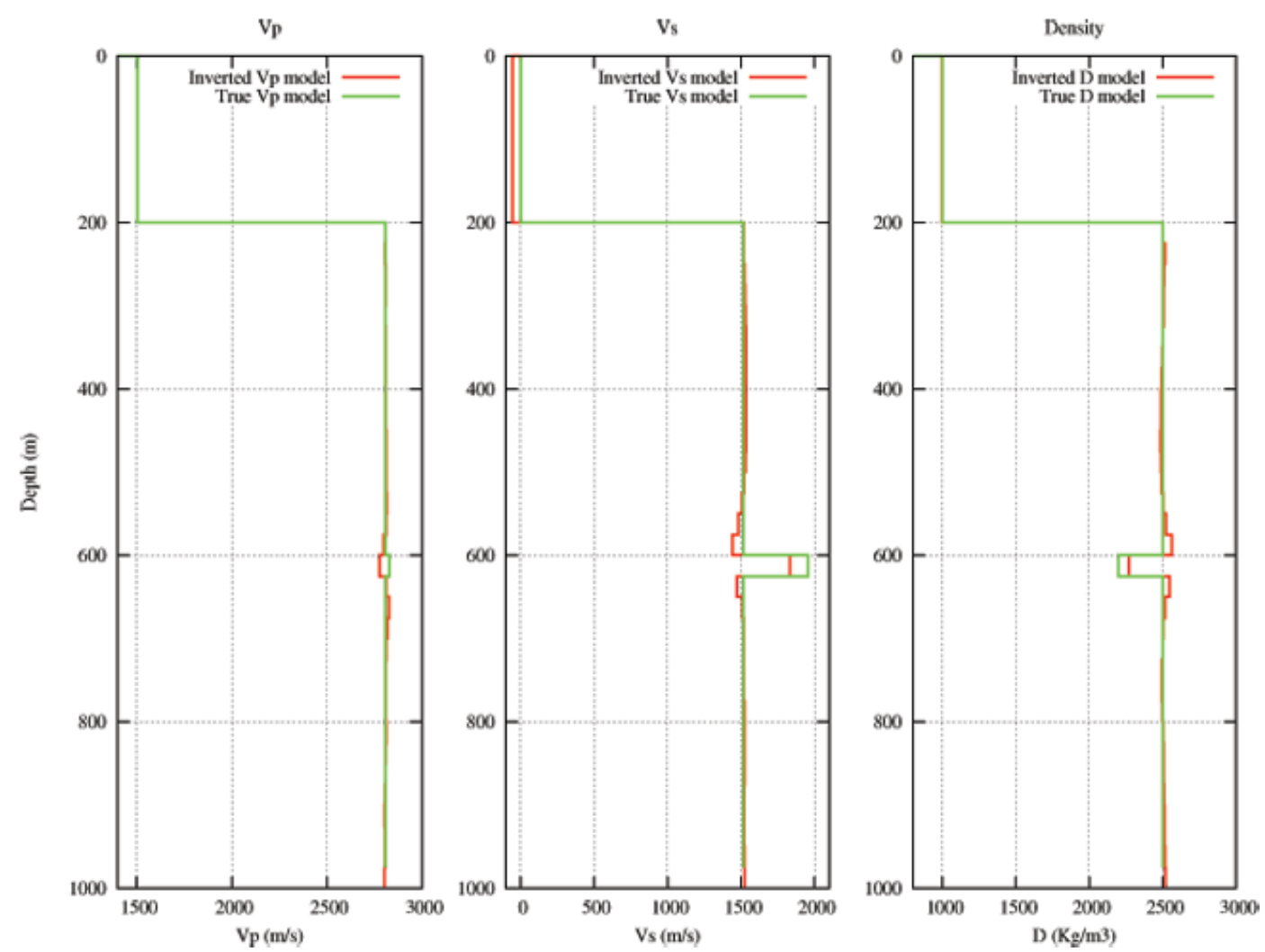

Figure 9 - Complete response inversion results for the $25 \mathrm{~m}$ sandstone model.

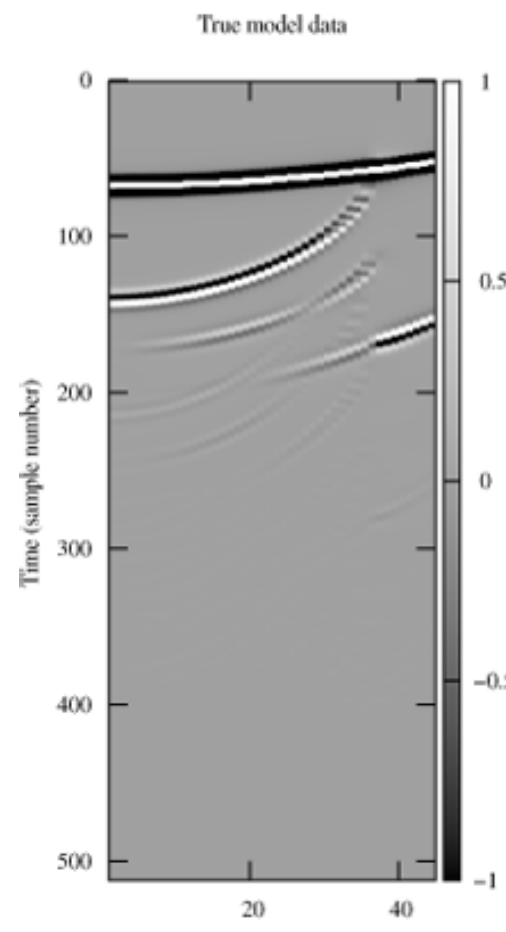

Ray parameter (sample number)

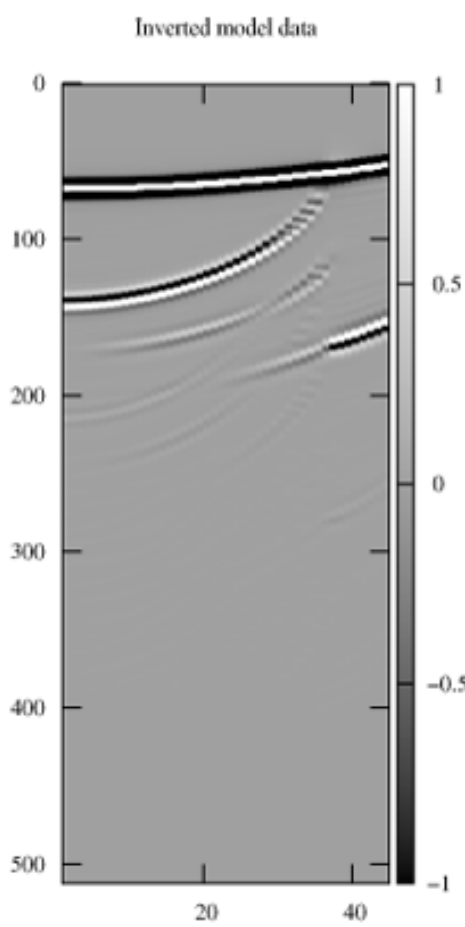

Ray parameter (sample number)

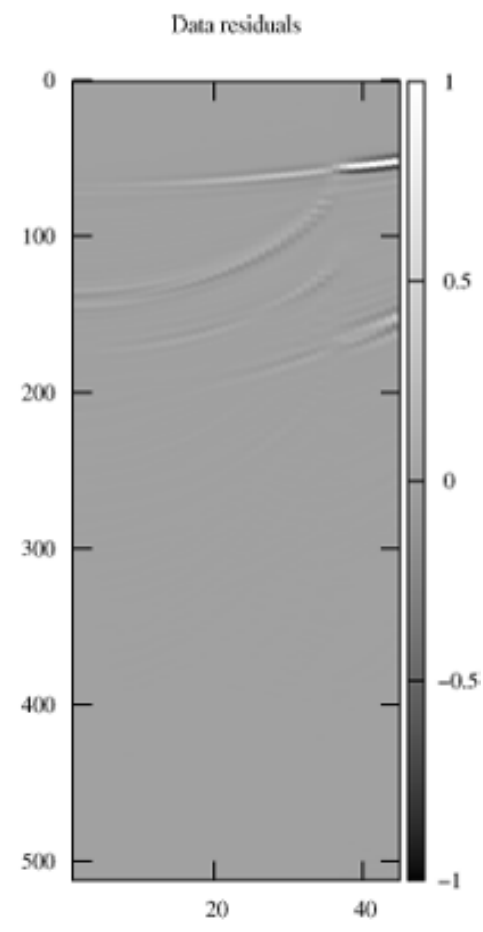

Ray parameter (sample number)

Figure 10 - Reference data; data from complete response inversion model; and data residuals for the $25 \mathrm{~m}$ sandstone model. 

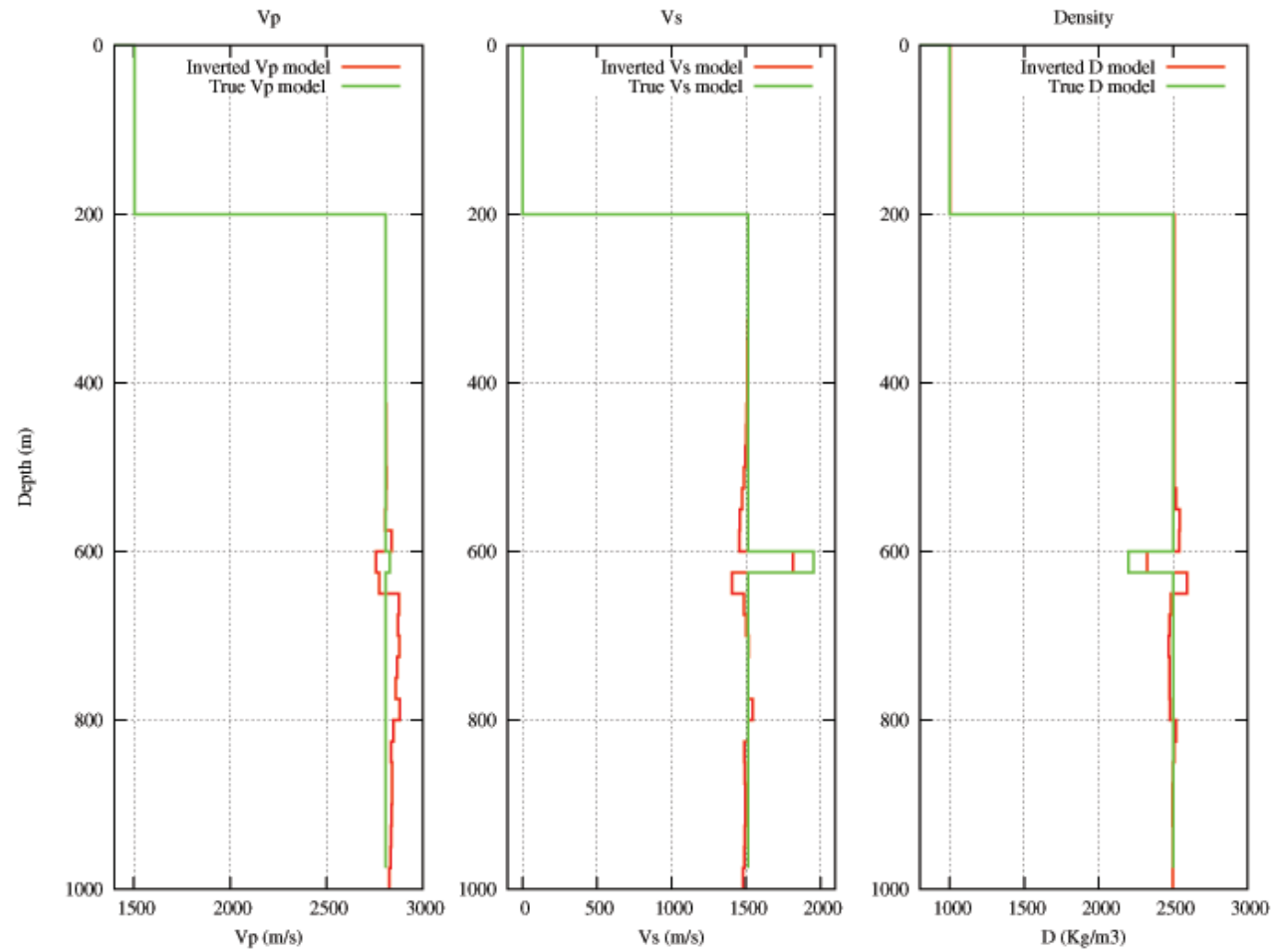

Figure 11 - Partial response inversion results for the $25 \mathrm{~m}$ sandstone model.

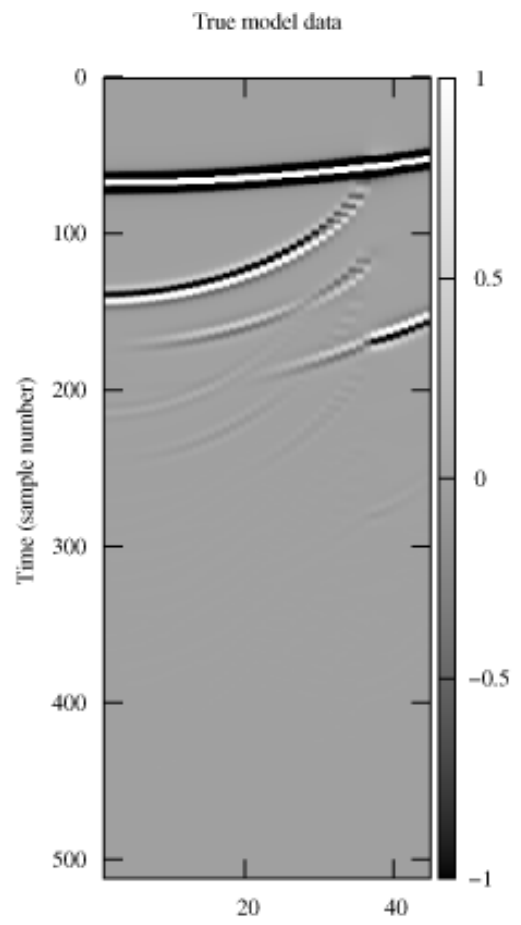

Ray parameter (sample number)

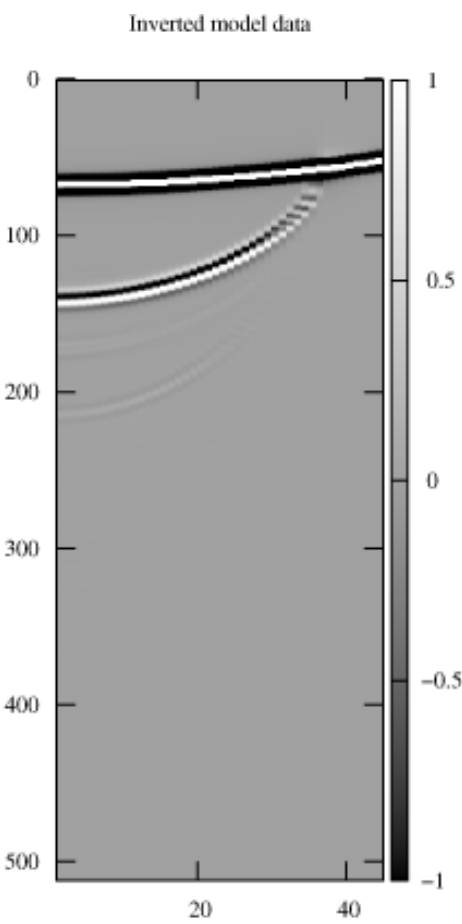

Ray parameter (sample number)

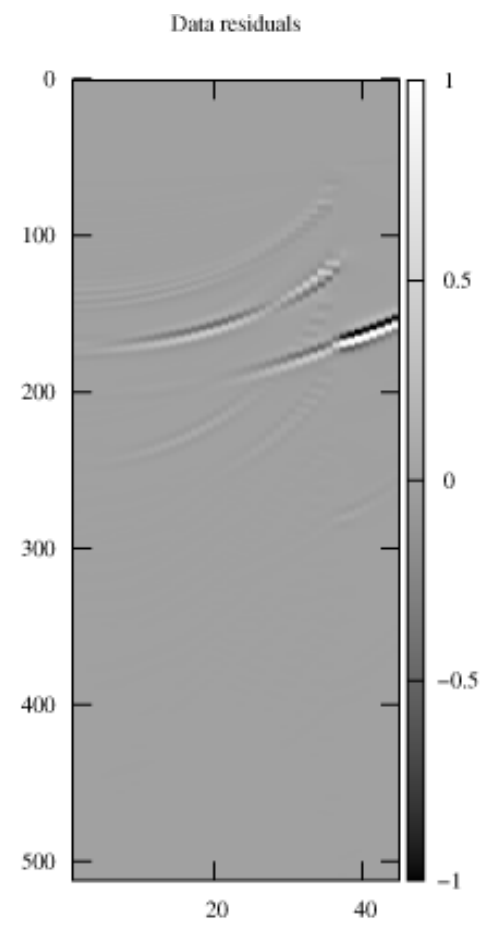

Ray parameter (sample number)

Figure 12 - Reference data; data from partial response inversion model; and data residuals for the $25 \mathrm{~m}$ sandstone model 

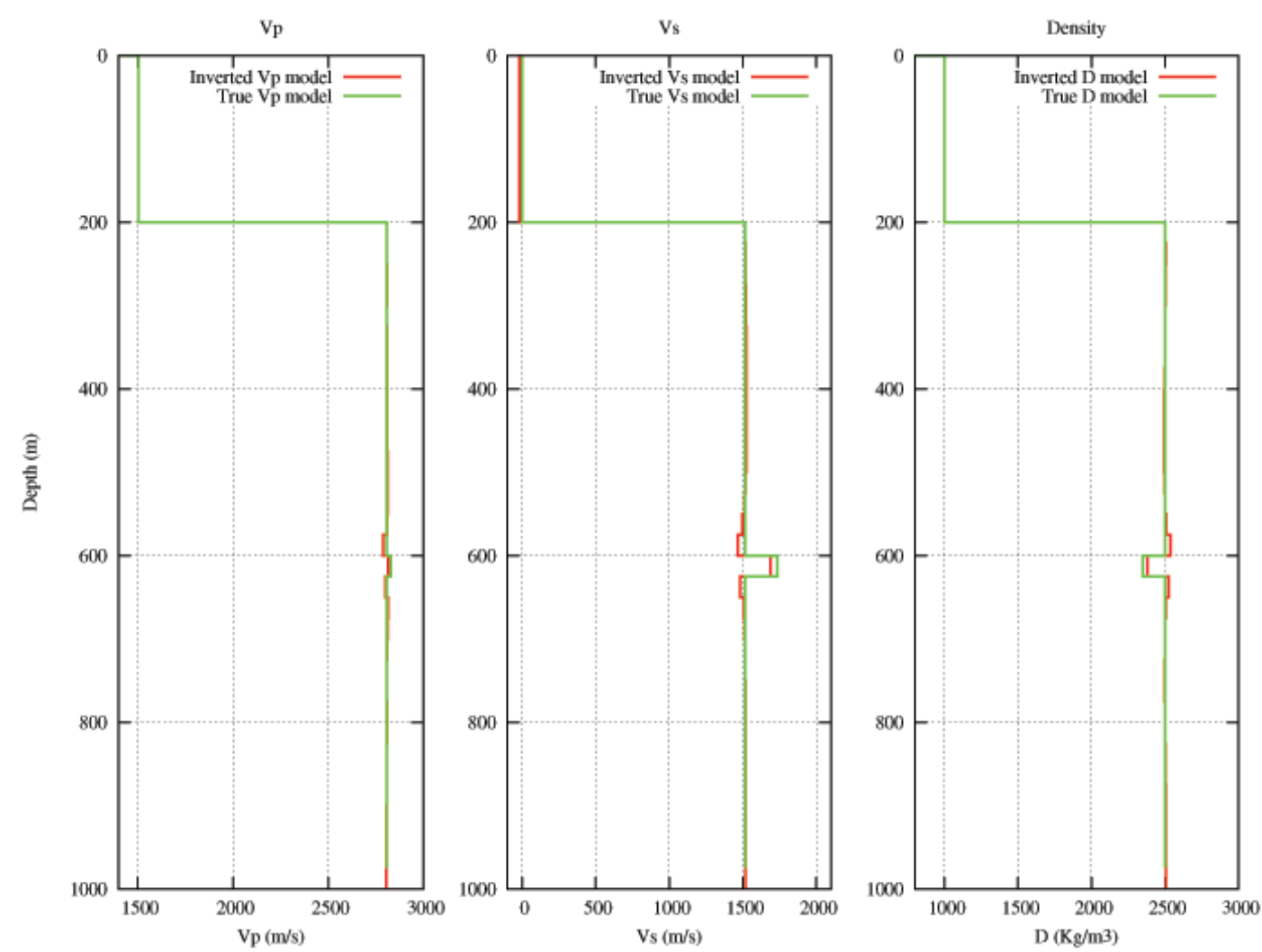

Figure 13 - Complete response inversion results for the $25 \mathrm{~m}$ reduced contrast sandstone model.

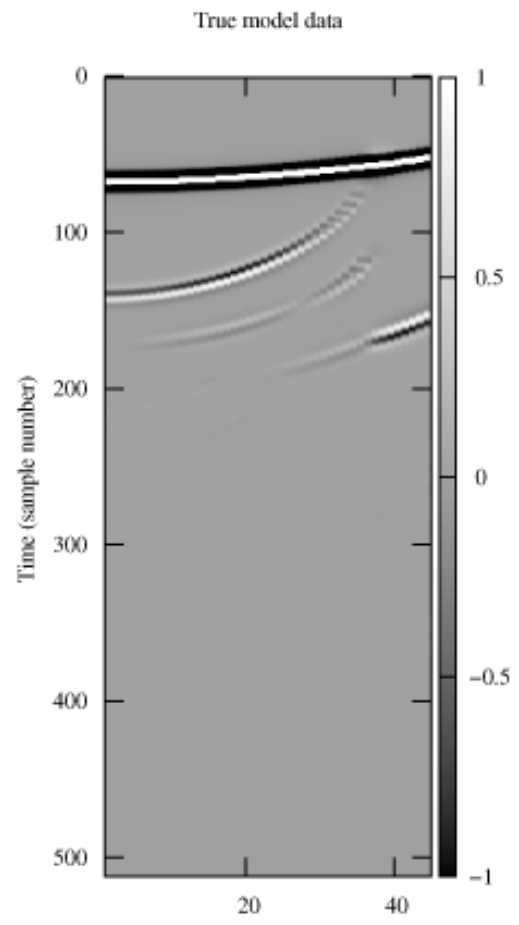

Ray parameter (sample number)

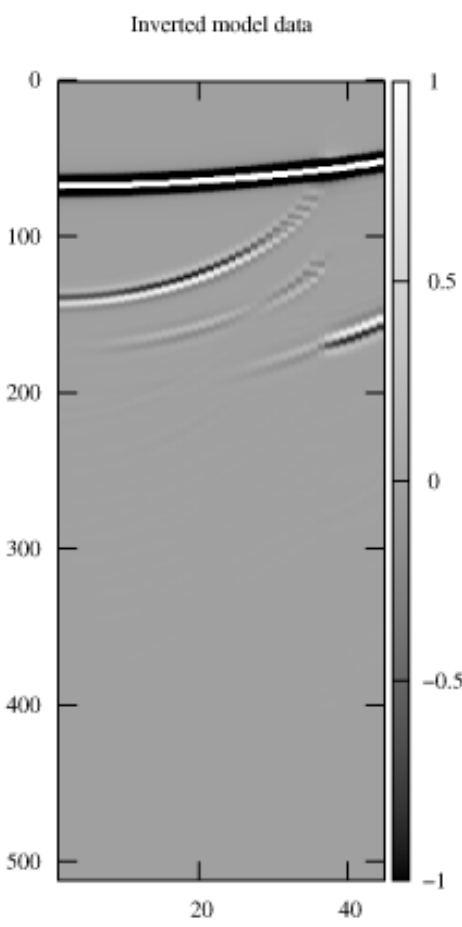

Ray parameter (sample number)

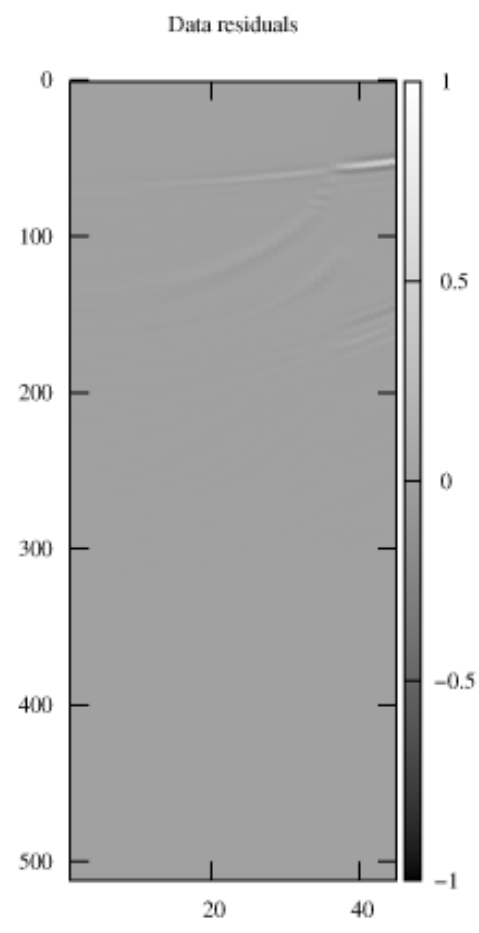

Ray parameter (sample number)

Figure 14 - Reference data; data from complete response inversion model; and data residuals for the $25 \mathrm{~m}$ reduced contrast sandstone model. 

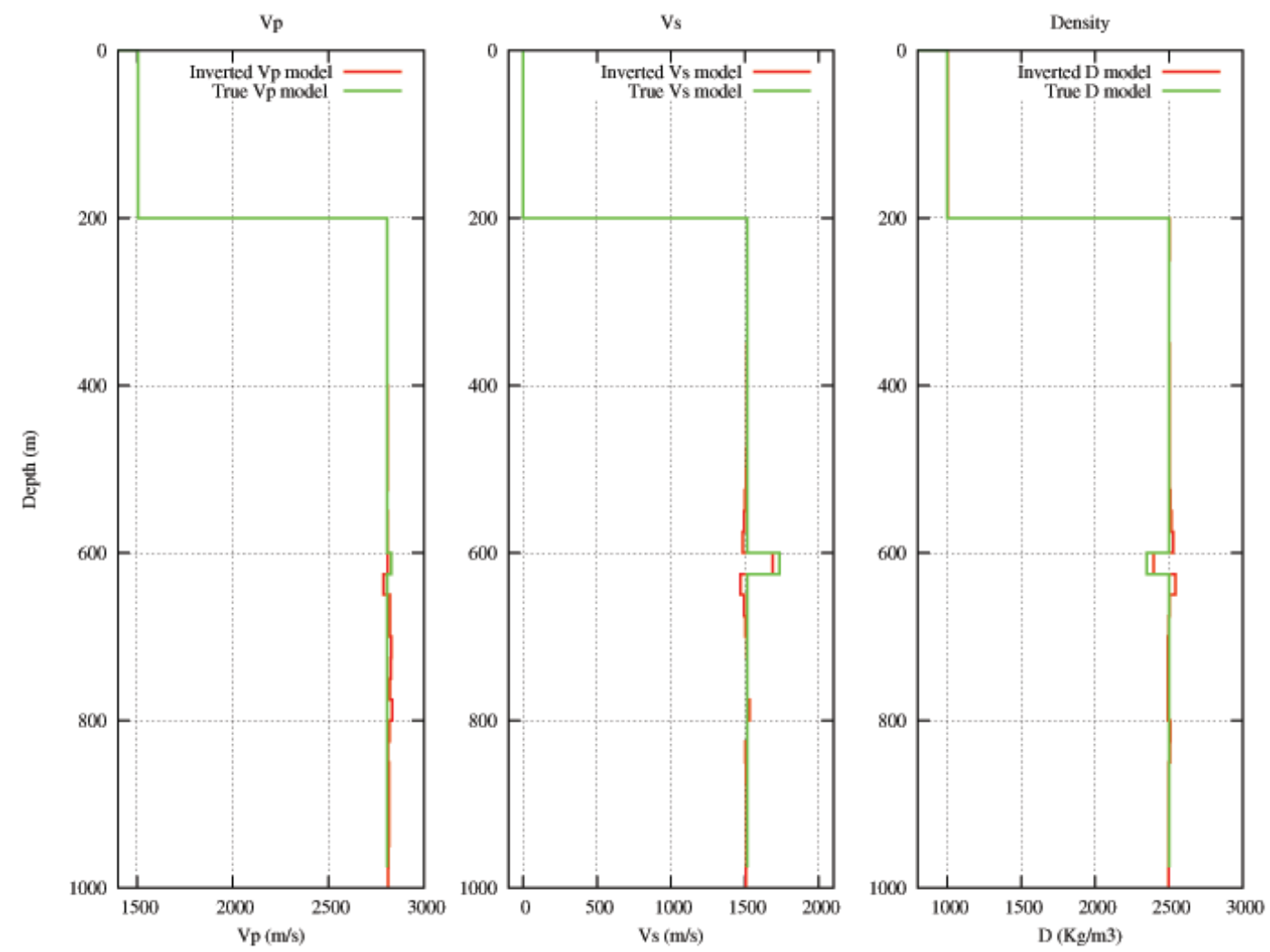

Figure 15 - Partial response inversion results for the $25 \mathrm{~m}$ reduced contrast sandstone model.

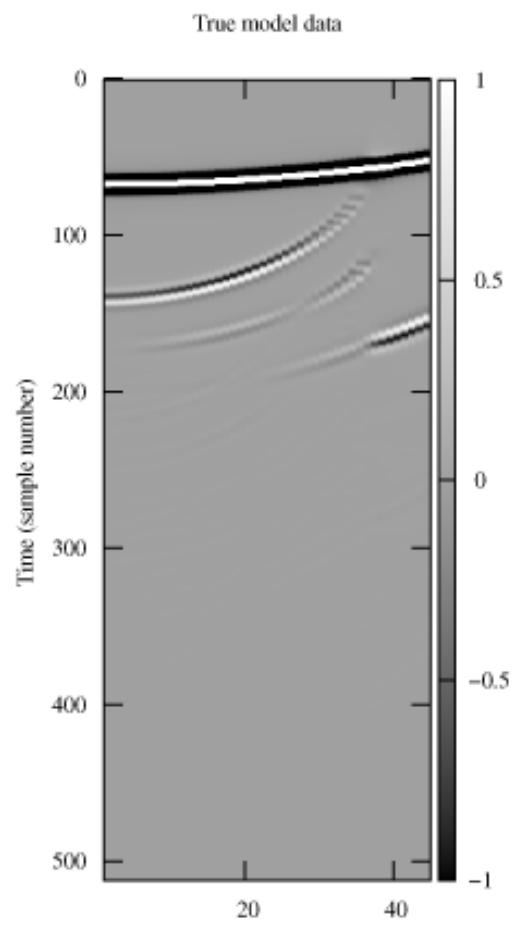

Ray parameter (sample number)

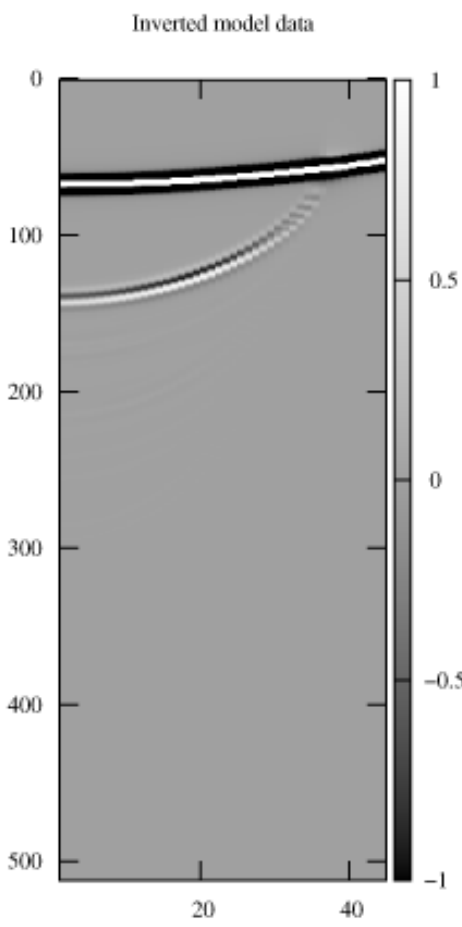

Ray parameter (sample number)

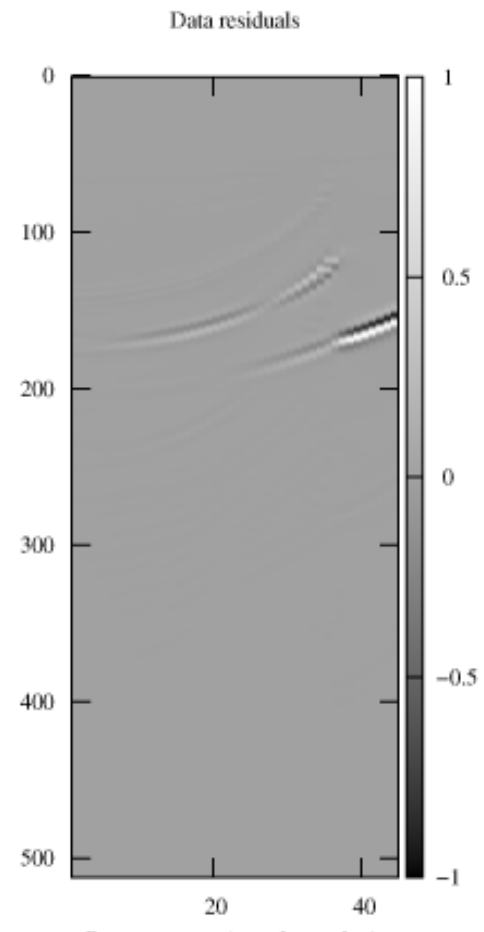

Ray parameter (sample number)

Figure 16 - Reference data; data from partial response inversion model; and data residuals for the $25 \mathrm{~m}$ reduced contrast sandstone model. 

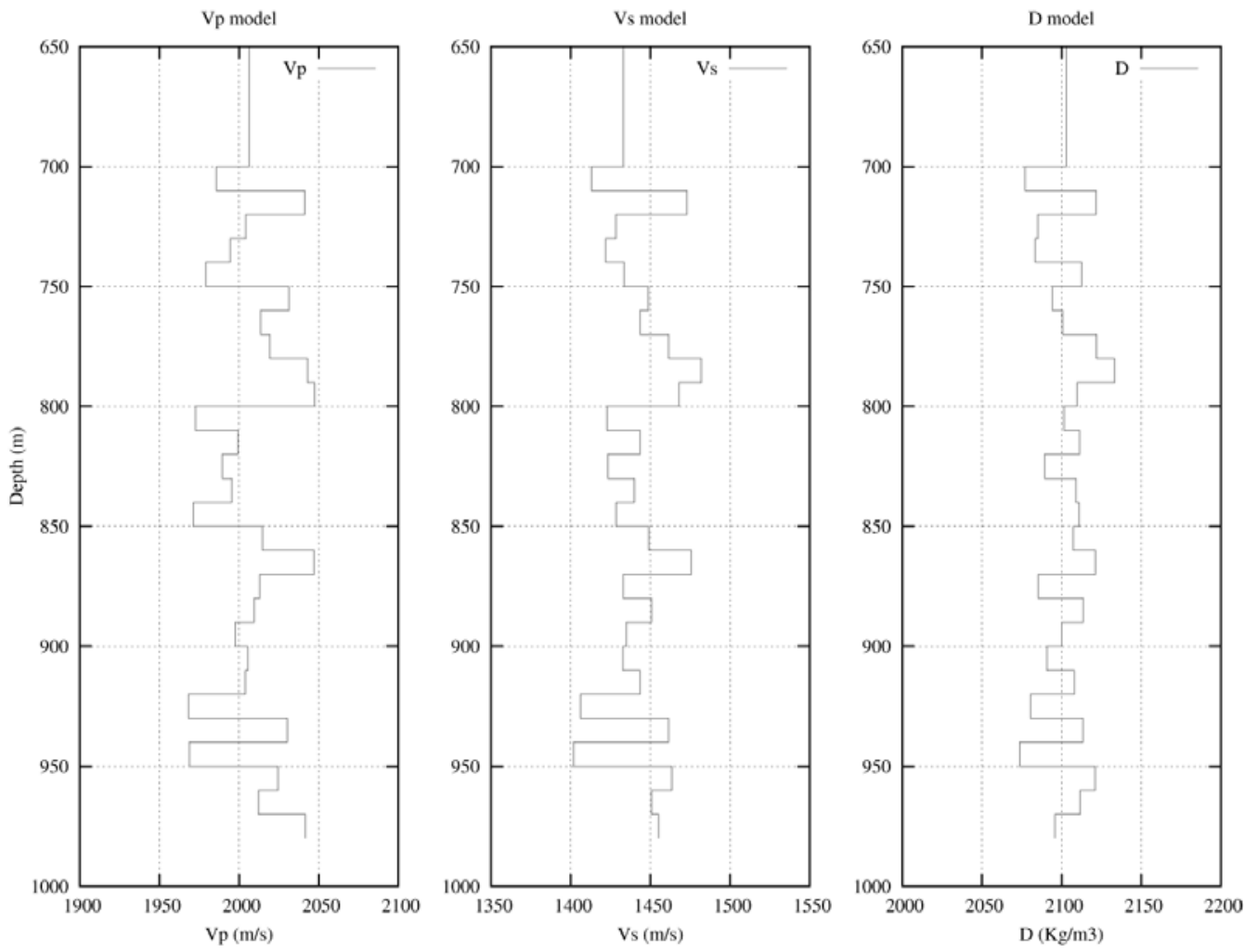

Figure $17-V p, V s$ and density models that represent a portion of a moderate contrast siliciclastic sedimentary section.
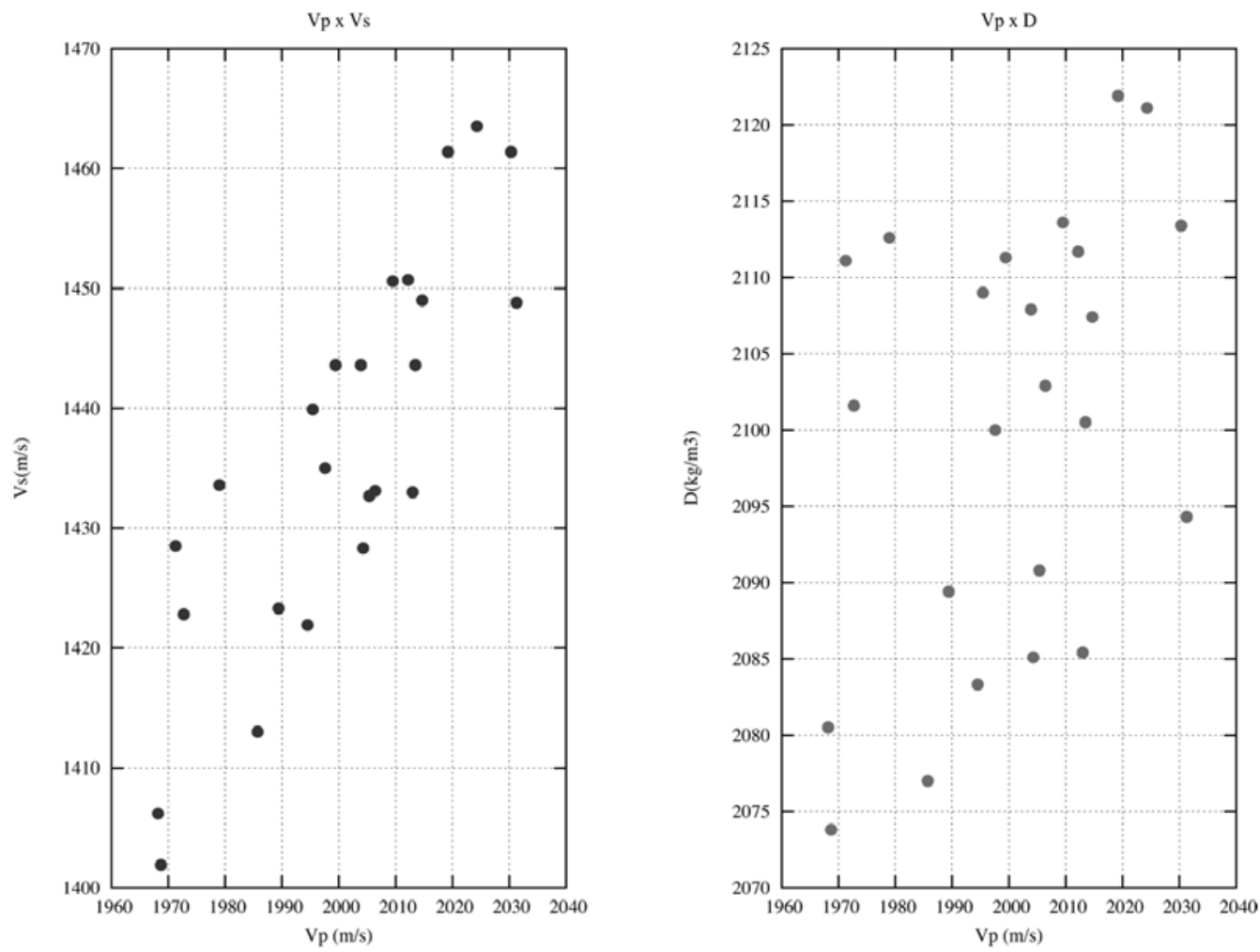

Figure 18 - Relationships between $V p$ and $V s$ and $V p$ and density of the model that represents a portion of a moderate contrast siliciclastic sedimentary section. 


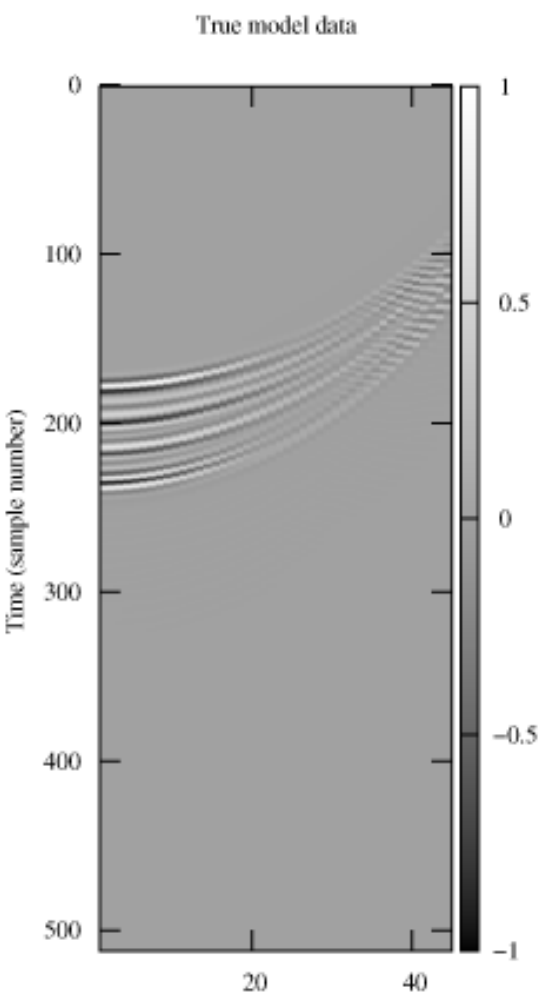

Ray parameter (sample number)

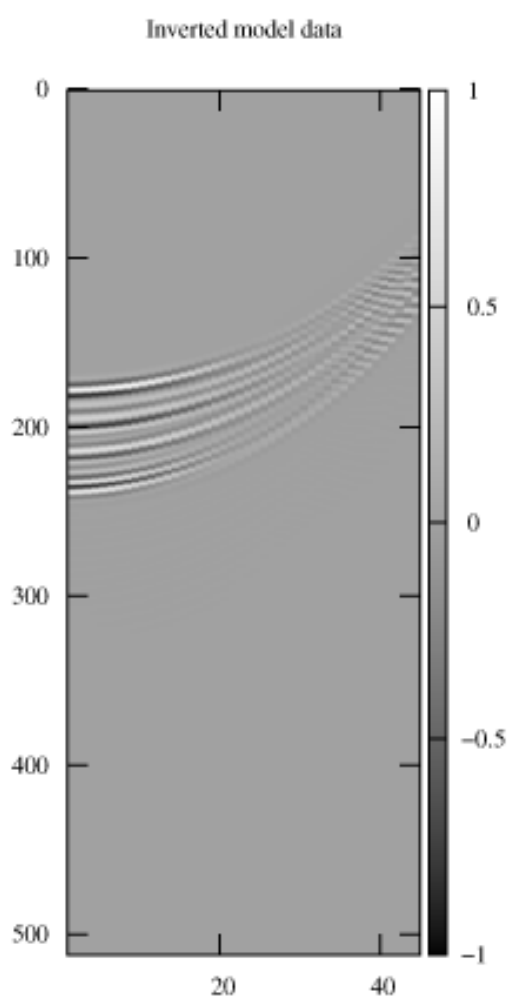

Ray parameter (sample number)

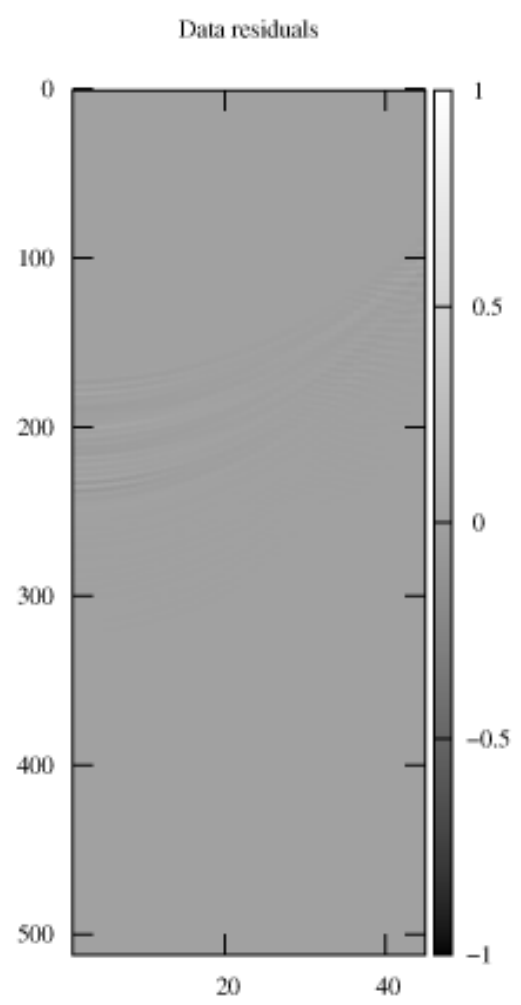

Ray parameter (sample number)

Figure 19 - Reference data; data from complete response inversion model; and data residuals for the moderate contrast siliciclastic model in Figure 17.

were recovered. The difference between the data from the complete response inversion model and the data calculated with the true model was minimal (Fig. 19). The plot of error in the data versus number of iterations of the inversion shows that little would be gained by further increasing the number of iterations of the inversion (Fig. 20). The results shown in Figure 21 were obtained with the complete response inversion.

AVO inversion was performed using the Aki \& Richards approximation, with the same data and initial models used in the waveform inversion; $V p, V s$ and density reflectivities were estimated and then integrated to estimate the properties of the layers. The AVO inversion was able to correctly recover the $V p$ and $V s$ features of the true model, but with less precision than the result obtained by the waveform inversion. The density model obtained from the AVO inversion, as expected, has lower quality in recovering the features and absolute values of the density profile. The results are shown in Figure 22.

These results show that the incorporation in the waveform inversion of propagation phenomena that are not considered by the convolutional model can improve the estimates of $V p, V s$, and especially density.

\section{Model of a siliciclastic sedimentary section with high contrasts of $V s$ and density}

The model used above was modified to evaluate the results of the waveform inversion when the layers have high $V s$ and density contrasts (Fig. 23), so that the difference between the complete response of the medium and the partial response, that disregards the conversions, is not negligible. Total and partial reflectivity responses, as well as the difference between total and partial responses (Fig. 24) were calculated with this model. Unlike the model of the previous example, where the difference between the inversion results using the partial or complete response is negligible, it is expected that the waveform inversion algorithm using the complete response of the medium will provide better results. The data used as input to both algorithms contains the complete response of the medium. In both cases, constant values representing mean values of $V p, V s$ and density were used as initial models.

Frequencies between $0-96 \mathrm{~Hz}$ and angles up to 45 degrees were used in the inversion. The results of waveform inversions using both complete and partial responses of the medium is shown in Figures 25 and 26, respectively. The waveform inver- 


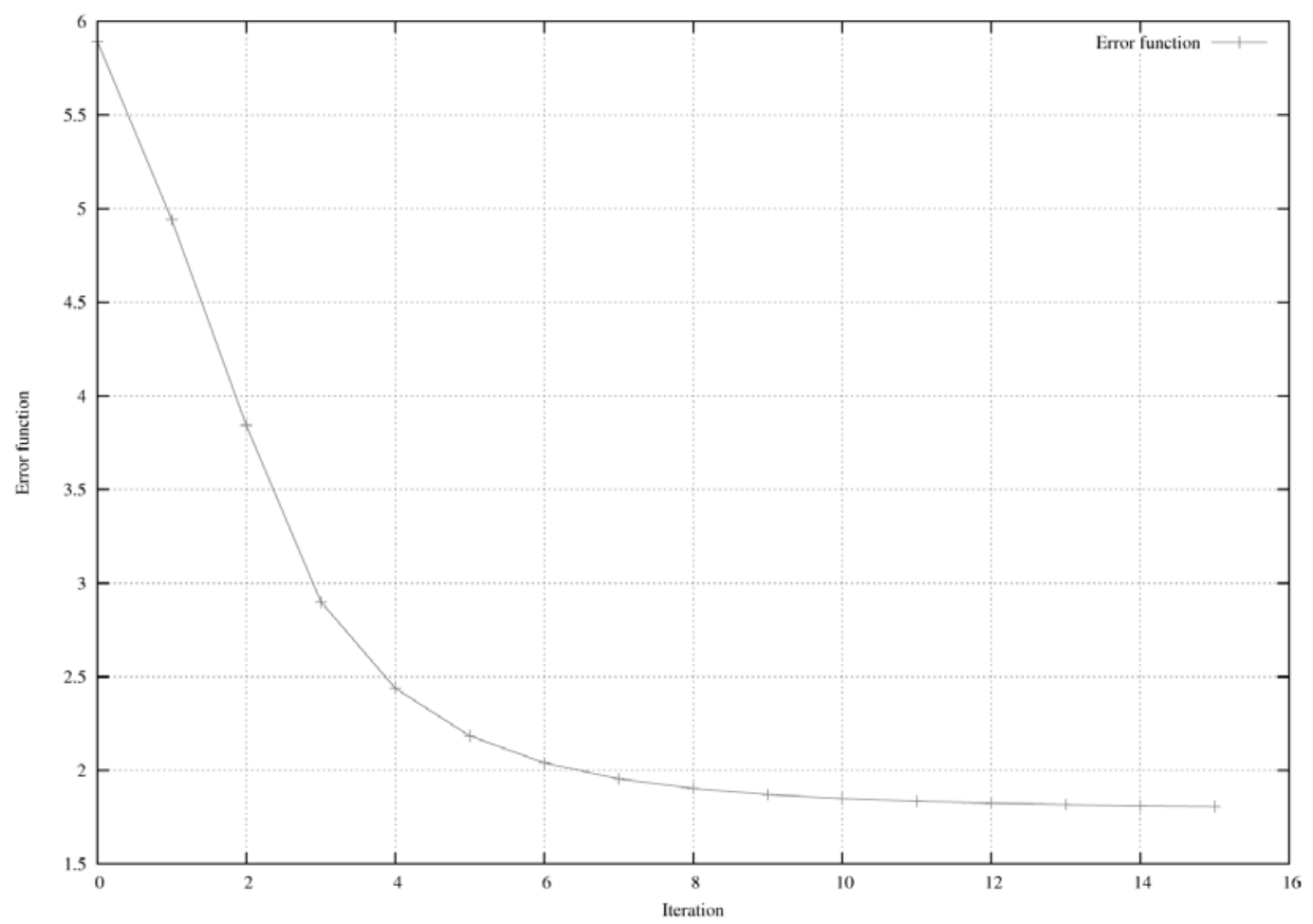

Figure 20 - Error function versus number of iterations for the complete response inversion of the data from the moderate contrast siliciclastic model.
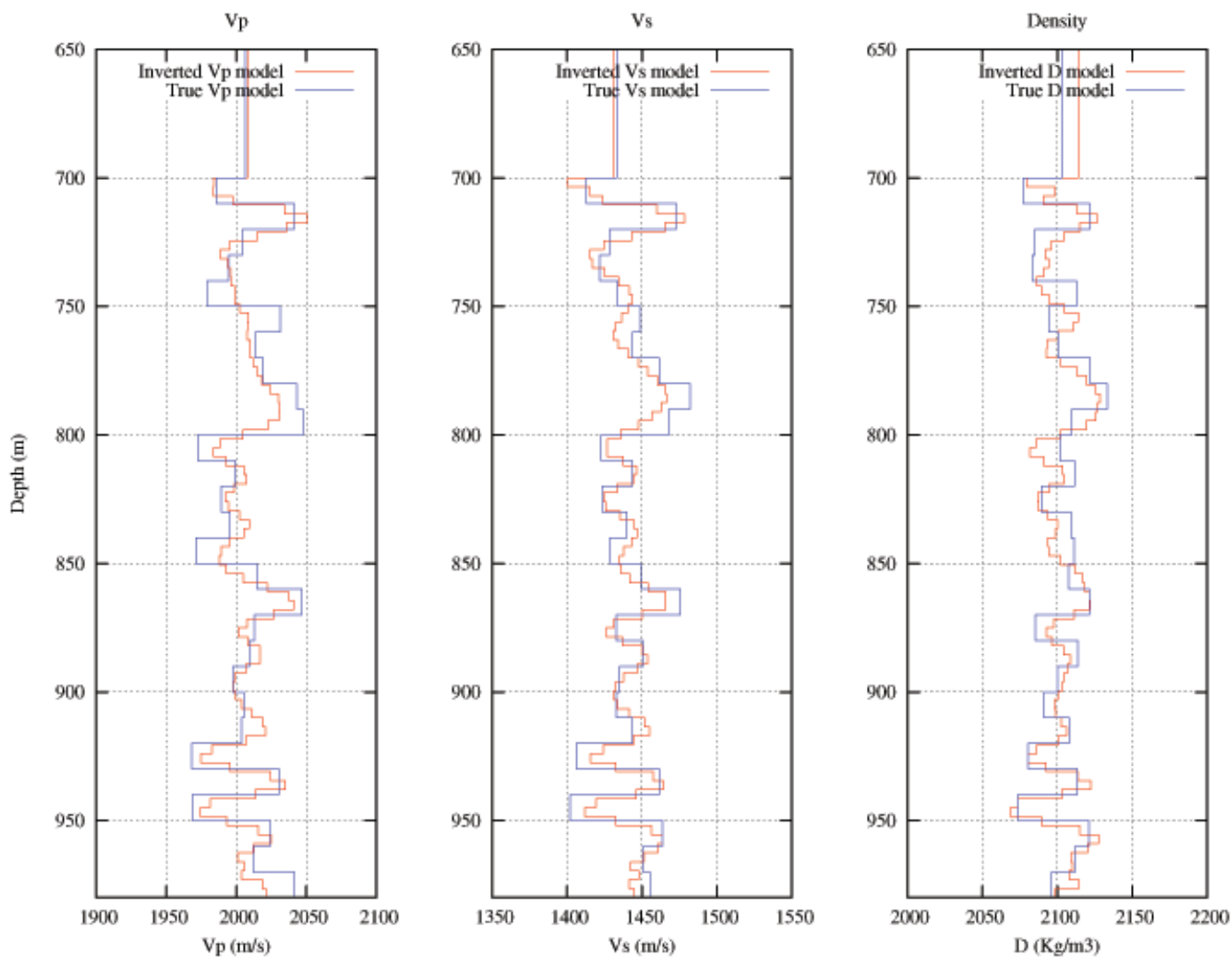

Figure 21 - Complete response inversion results for the moderate contrast siliciclastic model. 

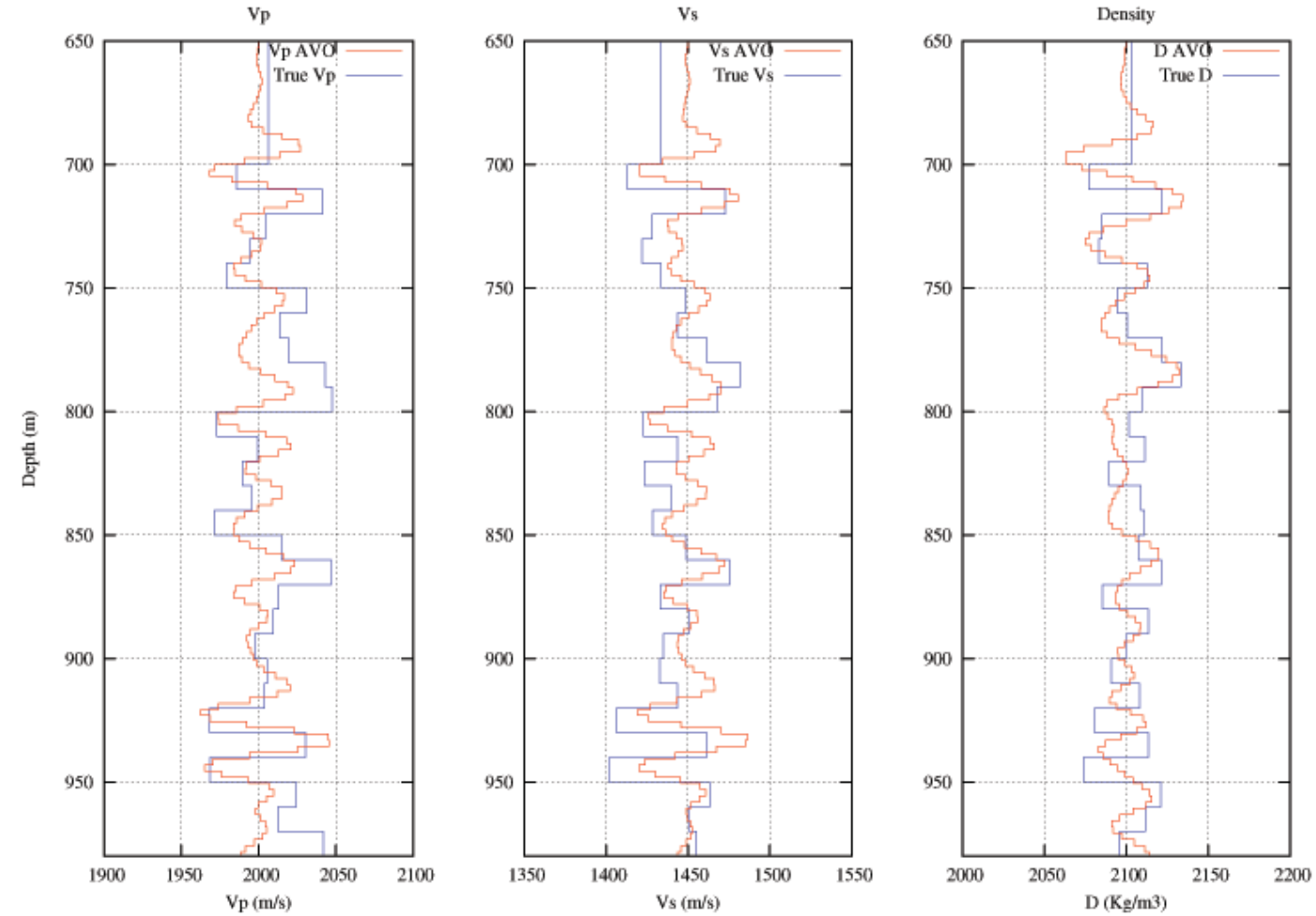

Figure 22 - AVO inversion results for the moderate contrast siliciclastic model.
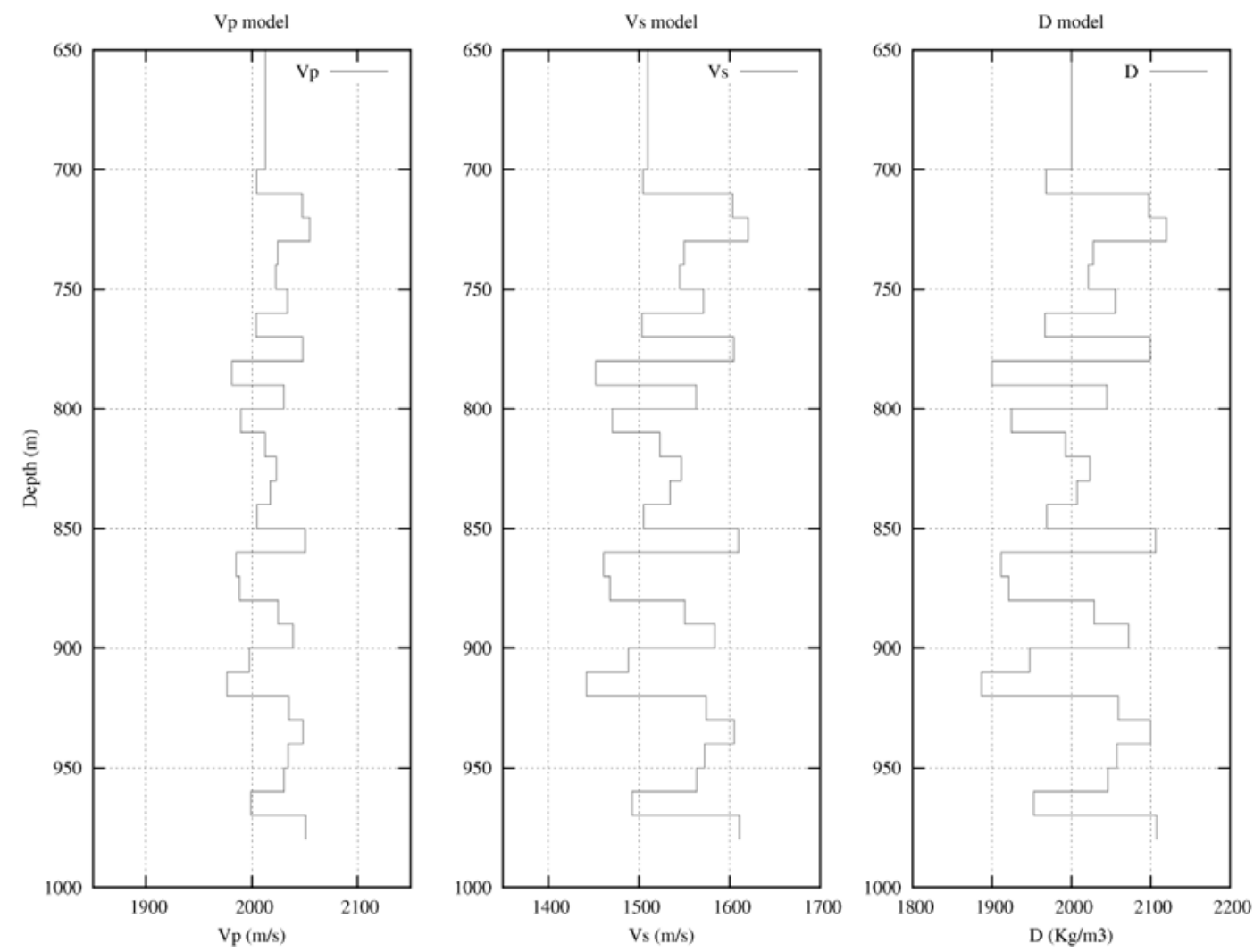

Figure $23-V p, V s$ and density models that represent a portion of a siliciclastic sedimentary section with high contrasts of $V s$ and density. 


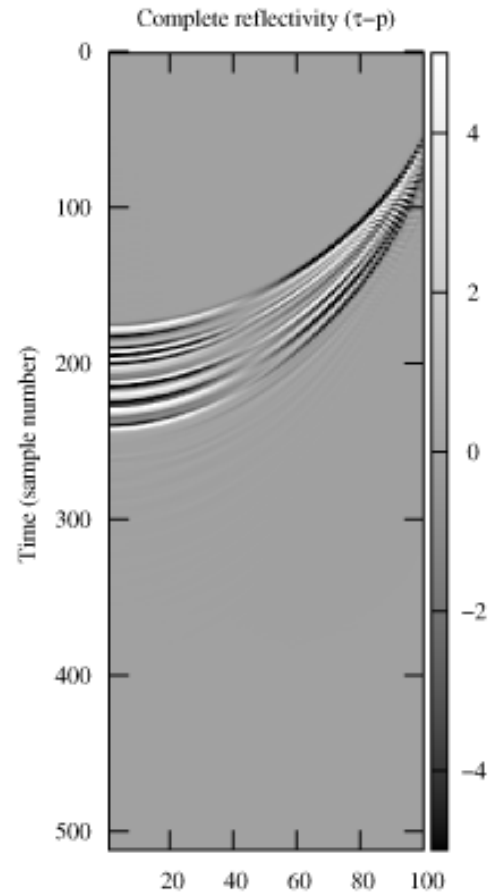

Ray parameter (sample number)

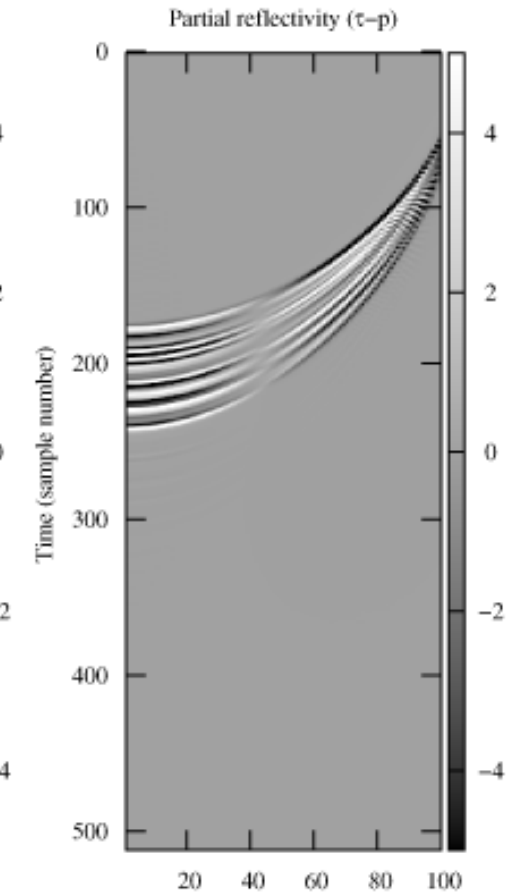

Ray parameter (sample number)

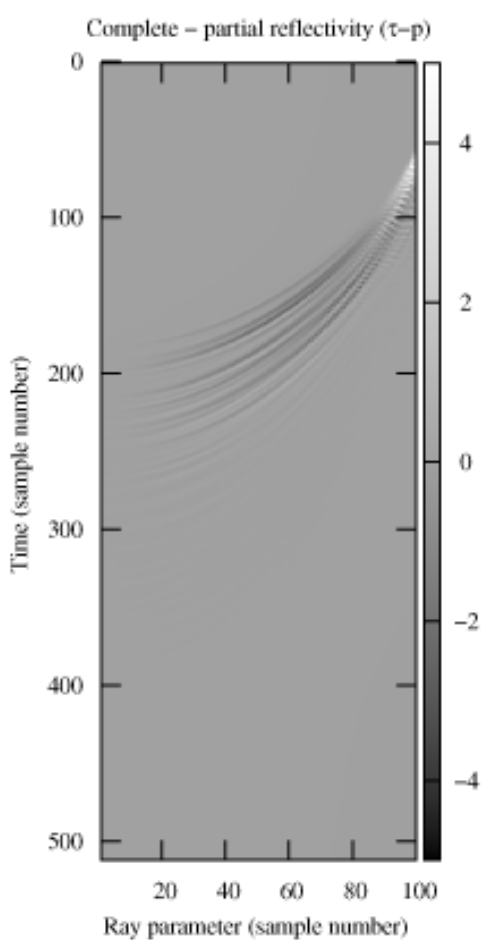

Ray parameter (sample number)

Figure 24 - Complete and partial responses of the high contrast siliciclastic model in Figure 23, and the difference between complete and partial responses.
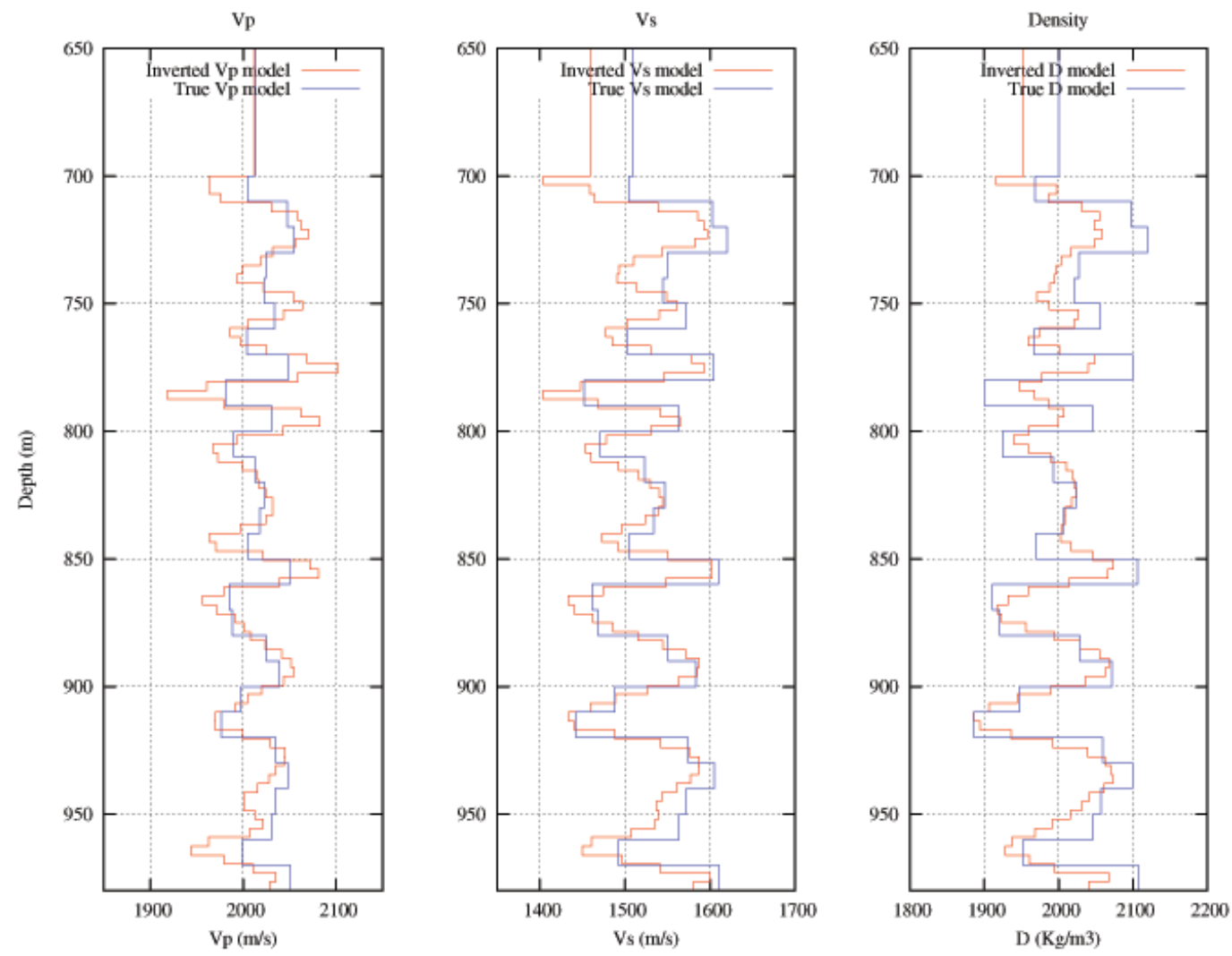

Figure 25 - Complete response inversion results for the high contrast siliciclastic model. 

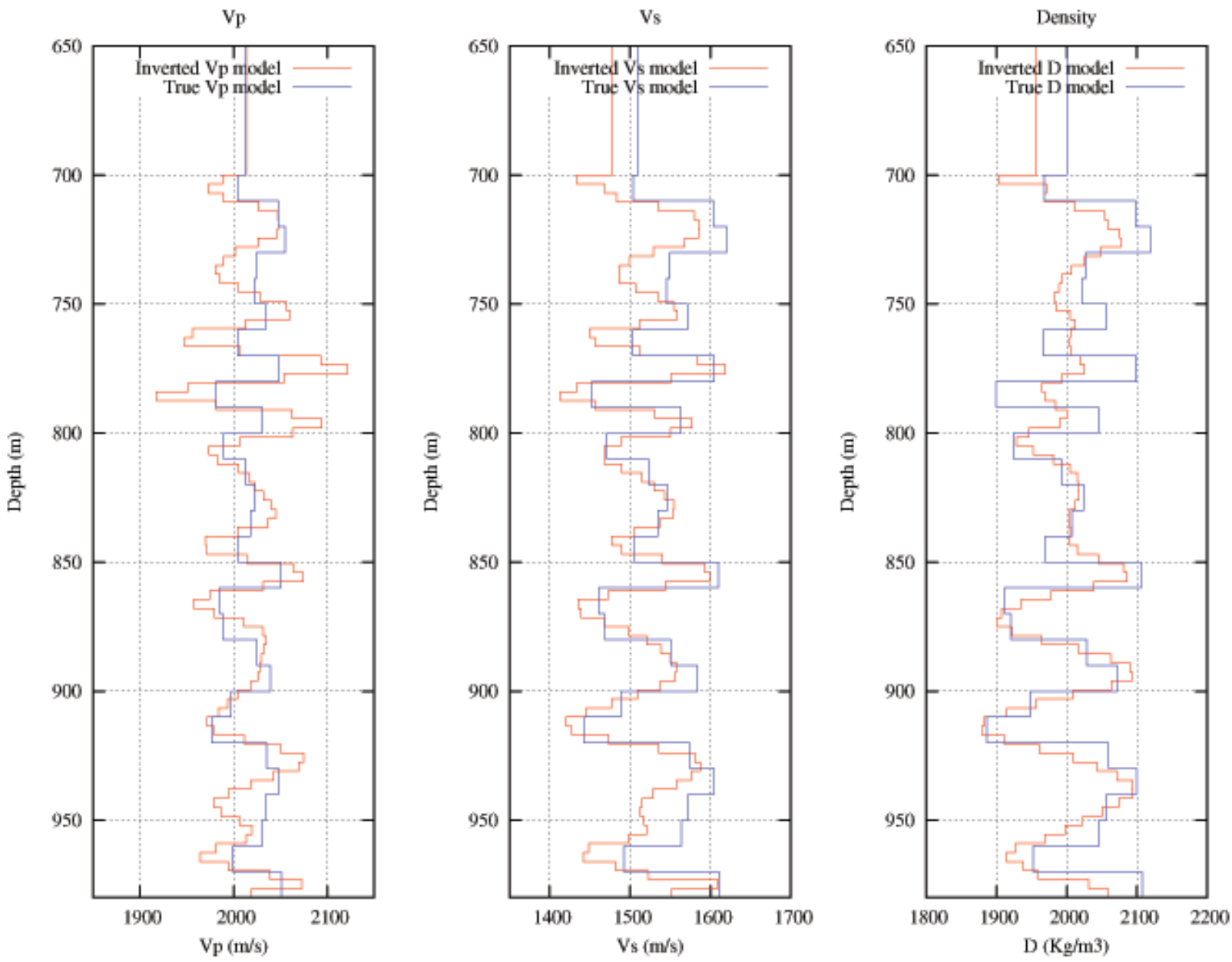

Figure 26 - Partial response inversion results for the high contrast siliciclastic model.

sion using the complete response has reached more reliable results in the recovery of $V p$ and density models, especially between 700 and $800 \mathrm{~m}$ depths. $V s$ models recovered by the two algorithms are very similar, both showing excellent correspondence with the true model. The data residuals of the complete response inversion (Fig. 27) is smaller than the data residuals of the partial response inversion (Fig. 28), showing the impossibility to model certain features of the wafefield when using the partial response of the medium.

Analyzing the inversion results as $I p, I s$ and density profiles, we find great similarity between the $I p$ and $I s$ models obtained with the two inversion algorithms (Figs. 29 and 30). This situation reflects the fact often observed, that the parameters best resolved in the inversion of compressional waves are $I p, I s$ and density in this order. We have seen by way of examples with synthetic data that density is better resolved when using the waveform inversion algorithm using the complete response of the medium.

\section{CONCLUSIONS}

The reflectivity method is appropriate both to evaluate the contribution of converted waves to compressional seismic data as to serve as a basis for elastic inversion algorithms that can take converted waves into account in the inversion.

Seismic modeling using the reflectivity method showed that converted waves can have a significant contribution to the amplitudes of compressional data. Furthermore, there are converted wave events that can be easily identified and filtered because of their lower velocity, and converted waves events that are difficult to identify and filter: locally converted waves whose transit time is very similar to compressional events.

The elastic inversion based on the reflectivity method is a reliable tool for estimating elastic properties of a layered medium, provided we have a velocity model that represents well enough the transit time of the primary reflections, in order to avoid cycleskipping. Tests with synthetic data showed very good results in 
True model data

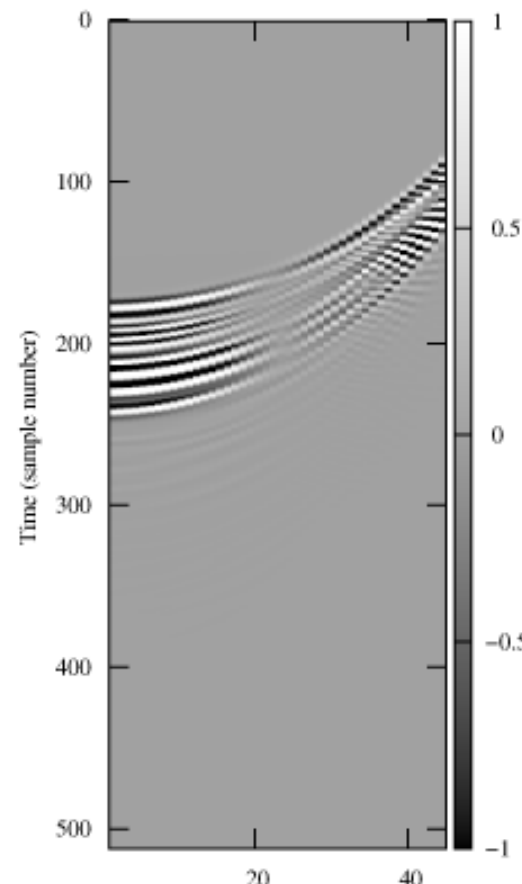

Ray parameter (sample number)

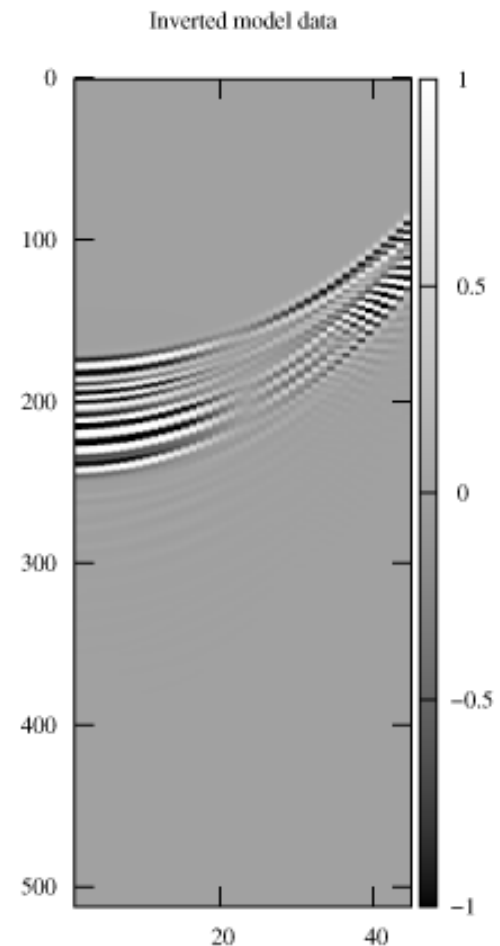

Ray parameter (sample number)
Data residuals

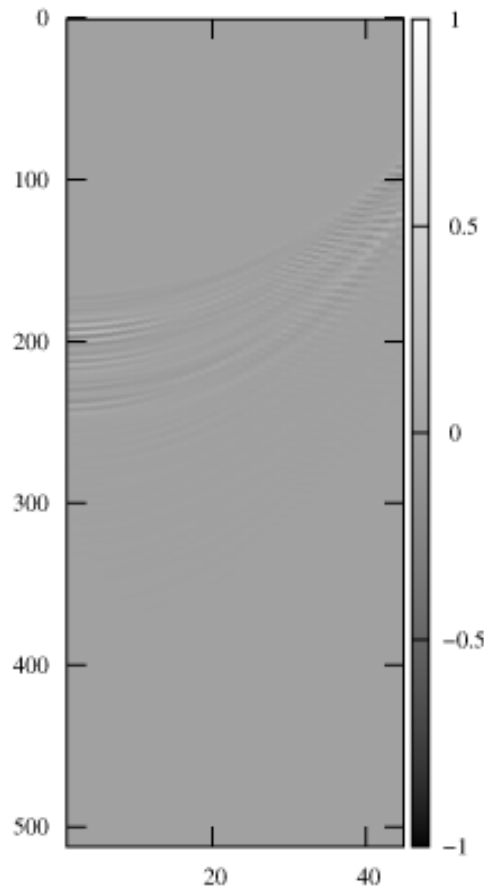

Ray parameter (sample number)

Figure 27 - Reference data; data from complete response inversion model; and data residuals for the high contrast siliciclastic model.

True model dati

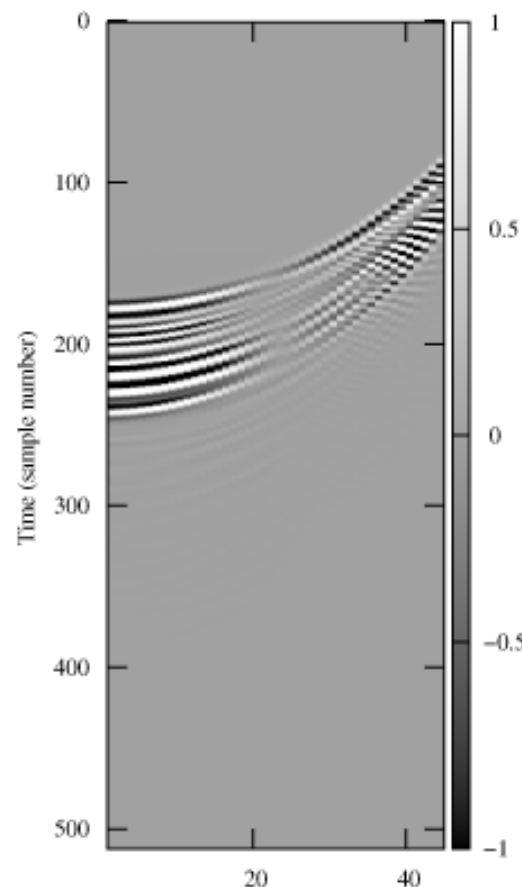

Ray parameter (sample number)
Inverted model data

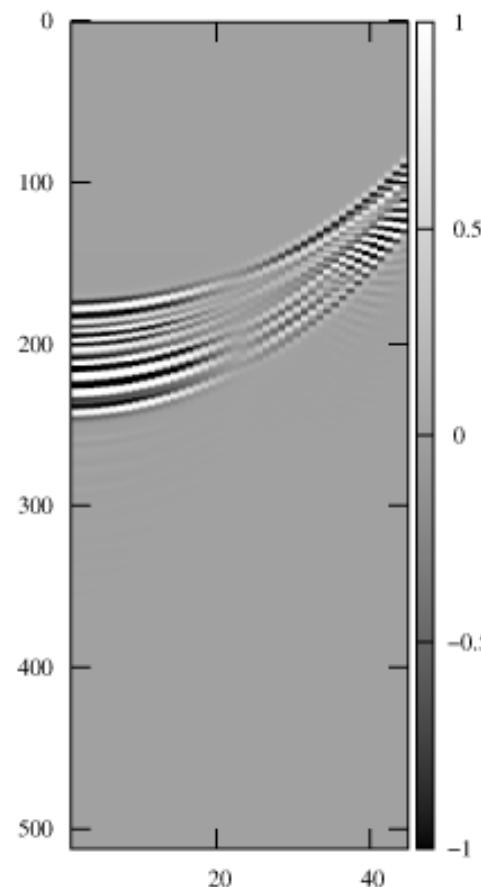

Ray parameter (sample number)
Data residuals

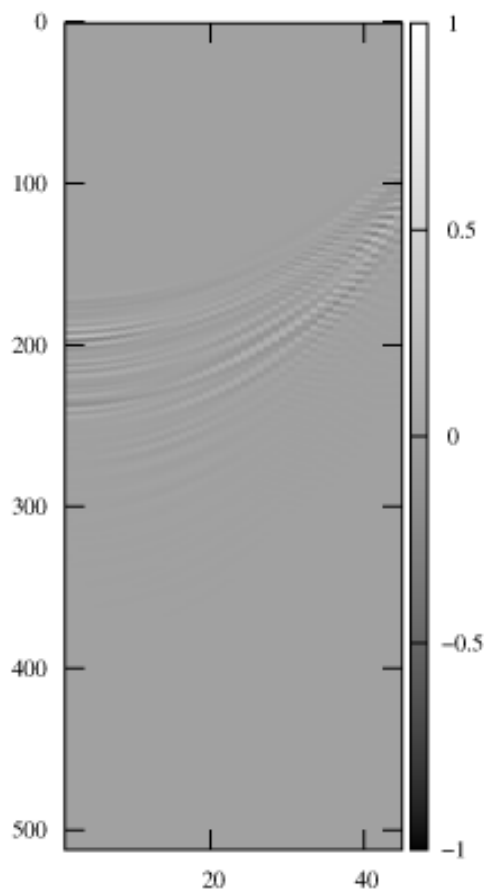

Ray parameter (sample number)

Figure 28 - Reference data; data from partial response inversion model; and data residuals for the high contrast siliciclastic model. 

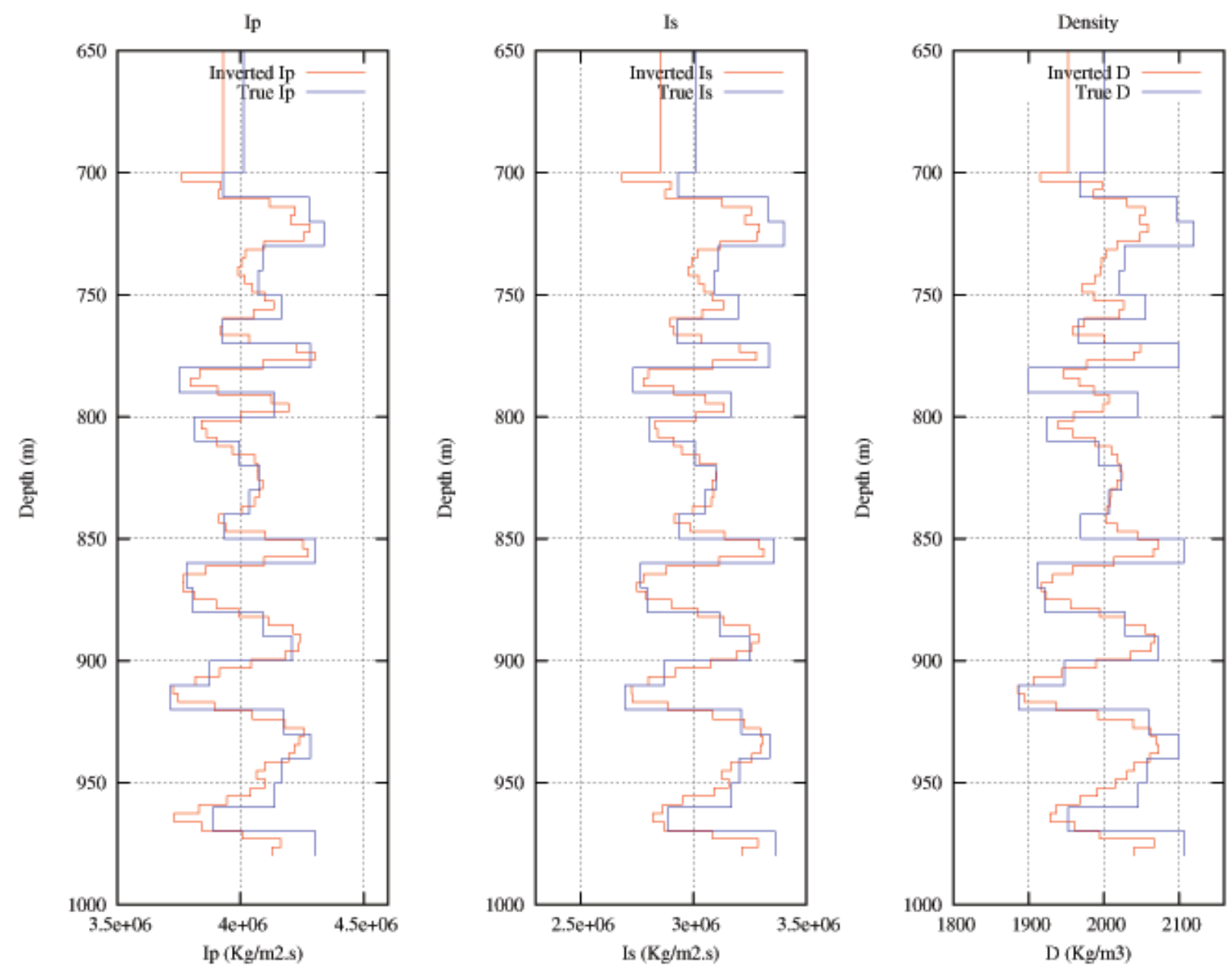

Figure 29 - Complete response inversion results for the high contrast siliciclastic model. Data are presented in terms of Ip, Is and density.

the estimation of $P$ and $S$ wave impedances. The density obtained with the waveform inversion showed good results.

The development of recursive algorithms for the calculation of partial derivatives of the reflectivity allowed us to calculate the Jacobian matrix accurately and efficiently. This allowed the use of the Levenberg-Marquardt algorithm and a quick convergence to minimize the error function, making the inversion method based on the reflectivity method more attractive from the computational point of view.

Waveform inversion using synthetic data showed good results, confirming the potential of waveform inversion to estimate with greater fidelity the elastic properties of the medium. In comparison with the AVO inversion, the waveform inversion achieved superior results, particularly in the estimated densities. Waveform inversion considering the partial response of the medium achieved similar results as that obtained with the waveform in- version considering the complete response, except in situations where the combination of thin layers with high contrasts in properties occurs. In these cases, the inversion results using the complete response is superior.

\section{APPENDIX A - Modeling using the reflectivity method}

The reflectivity method is applied in the plane-wave domain, parameterized by the angular frequency and ray parameter ( $w$ - $p$ domain) and considers that the earth can be locally approximated by a 1-D stratified medium. It is used to compute the complete seismic response of the medium, that is, considering transmission effects, multiples and conversions of all types occurring in layers as shown by Müller (1985); but it is also possible to derive expressions for calculating the medium response without taking into account certain events, for instance, $P S$ conversions. 

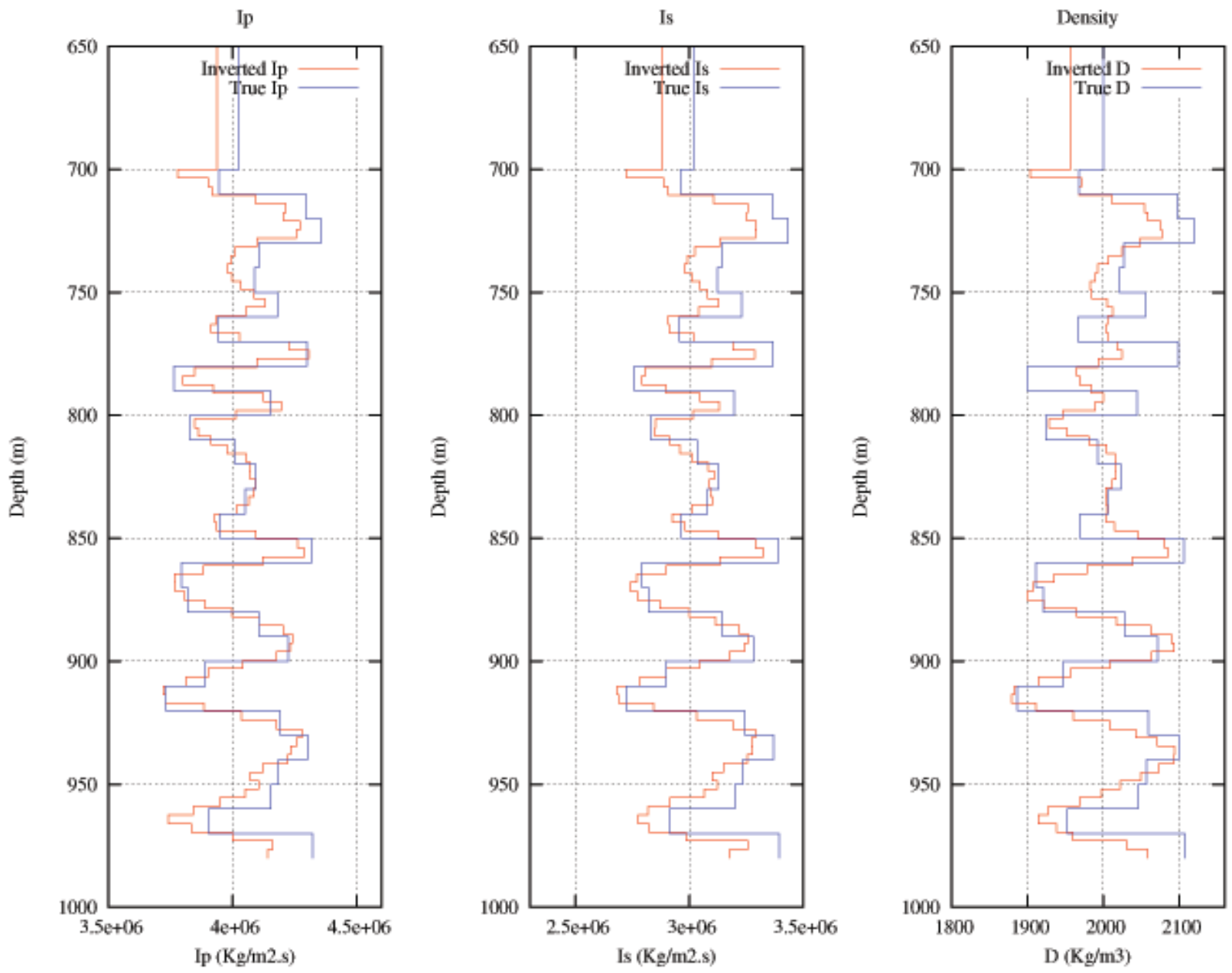

Figure 30 - Partial response inversion results for the high contrast siliciclastic model. Data are presented in terms of $I p, I s$ and density.

In the reflectivity method the response of a layered medium to a plane harmonic wave is built recursively from the last (deeper) to the first interface; the medium is considered to be limited above and below by a homogeneous half-space medium. A recursive expression for obtaining the full response is presented below:

$$
\mathbf{M}_{i}^{b}=\mathbf{R}_{i+1}^{d}+\mathbf{T}_{i+1}^{u}\left[\mathbf{I}-\mathbf{M}_{i+1}^{t} \mathbf{R}_{i+1}^{u}\right]^{-1} \mathbf{M}_{i+1}^{t} \mathbf{T}_{i+1}^{d} .
$$

where $\mathbf{M}_{i}^{t}$ and $\mathbf{M}_{i}^{b}$ are the reflectivity matrices at the top and bottom, respectively, of the ith layer

$$
\mathbf{M}_{i}^{t}=\left(\begin{array}{cc}
P P_{i}^{t} & S P_{i}^{t} \\
P S_{i}^{t} & S S_{i}^{t}
\end{array}\right) \quad \mathbf{M}_{i}^{b}=\left(\begin{array}{cc}
P P_{i}^{b} & S P_{i}^{b} \\
P S_{i}^{b} & S S_{i}^{b}
\end{array}\right)
$$

these matrices are related by the phase shift matrix $\mathbf{E}$ :

$$
\begin{gathered}
\mathbf{M}_{i}^{t}=\mathbf{E}_{i} \mathbf{M}_{i}^{b} \mathbf{E}_{i} ; \\
\mathbf{E}_{i}=\left(\begin{array}{cc}
e^{-i K_{z i} d_{i}} & 0 \\
0 & e^{-i K_{z i}^{\prime} d_{i}}
\end{array}\right) .
\end{gathered}
$$


The $\mathbf{T}_{i+1}^{u / d}$ and $\mathbf{R}_{i+1}^{u / d}$ matrices contains the ascending an descending transmission/reflection coefficients of the $i+1$ interface:

$$
\mathbf{R}_{i}^{u / d}=\left(\begin{array}{cc}
R_{P P_{i}}^{u / d} & R_{S P_{i}}^{u / d} \\
R_{P S_{i}}^{u / d} & R_{S S_{i}}^{u / d}
\end{array}\right) \quad \mathbf{T}_{i}^{u / d}=\left(\begin{array}{cc}
T_{P P_{i}}^{u / d} & T_{S P_{i}}^{u / d} \\
T_{P S_{i}}^{u / d} & T_{S S_{i}}^{u / d}
\end{array}\right) .
$$

If the mode conversions that occurs within the layers are disregarded, the reflectivity can be calculated by the equation given below, which can be seen as the scalar equivalent of Equation (1) and models only the $P P$ events (see Oliveira, 2009):

$$
r_{p p}^{i}=\left[\frac{R_{i}^{d}+r_{p p}^{i+1}\left(T_{i}^{d} T_{i}^{u}-R_{i}^{d} R_{i}^{u}\right)}{1-r_{p p}^{i+1} R_{i}^{u}}\right] Z_{i}^{-2},
$$

where $Z_{i}=e^{i k_{i} d_{i}}$. These recursive expressions allow us to obtain the seismic response of the stratified medium in the plane wave domain, that is, to obtain $r_{p p}^{0}(\omega, p)$ taking into account only $P$ waves and its reverberations as in Equation (3) or taking into account the complete response using Equation (1).

\section{APPENDIX B - Analytical method for calculating the Jacobian matrix}

The Levenberg-Marquardt algorithm requires the Jacobian, the matrix whose elements represent the derivatives of the wavefield with respect to the elastic parameters of the layers:

$$
\frac{\partial r_{p p}^{0}}{\partial \alpha_{n}}, \frac{\partial r_{p p}^{0}}{\partial \beta_{n}}, \frac{\partial r_{p p}^{0}}{\partial \rho_{n}} .
$$

Where $\alpha_{n}, \beta_{n}$ and $\rho_{n}$ represent respectively the $P$ and $S$ wave velocity and density of the $n t h$ layer.

We have developed a novel method for calculating such derivatives without the need for numerical approximations in an efficient manner. For this we derive Equation (1) with respect to the elastic parameters. Starting with $\alpha_{n}$, we obtain:

$$
\begin{gathered}
\frac{\partial \mathbf{M}_{i}^{b}}{\partial \alpha_{n}}=\frac{\partial \mathbf{R}_{i+1}^{d}}{\partial \alpha_{n}} \frac{\partial \mathbf{T}_{i+1}^{u}}{\partial \alpha_{n}}\left[\mathbf{I}-\mathbf{M}_{i+1}^{t} \mathbf{R}_{i+1}^{u}\right]^{-1} \mathbf{M}_{i+1}^{t} \mathbf{T}_{i+1}^{d}+ \\
+\mathbf{T}_{i+1}^{u}\left[\mathbf{I}-\mathbf{M}_{i+1}^{t} \mathbf{R}_{i+1}^{u}\right]^{-1}\left[\frac{\partial \mathbf{M}_{i+1}^{t}}{\partial \alpha_{n}} \mathbf{R}_{i+1}^{u}+\mathbf{M}_{i+1}^{t} \frac{\partial \mathbf{R}_{i+1}^{u}}{\partial \alpha_{n}}\right]\left[\mathbf{I}-\mathbf{M}_{i+1}^{t} \mathbf{R}_{i+1}^{u}\right]^{-1} \mathbf{M}_{i+1}^{t} \mathbf{T}_{i+1}^{d}+ \\
+\mathbf{T}_{i+1}^{u}\left[\mathbf{I}-\mathbf{M}_{i+1}^{t} \mathbf{R}_{i+1}^{u}\right]^{-1} \frac{\partial \mathbf{M}_{i+1}^{t}}{\partial \alpha_{n}} \mathbf{T}_{i+1}^{d}+\mathbf{T}_{i+1}^{u}\left[\mathbf{I}-\mathbf{M}_{i+1}^{t} \mathbf{R}_{i+1}^{u}\right]^{-1} \mathbf{M}_{i+1}^{t} \frac{\partial \mathbf{T}_{i+1}^{d}}{\partial \alpha_{n}}
\end{gathered}
$$

To obtain this equation the following derivation rules of a matrix $\mathbf{A}$, whose elements are functions of one variable, were used; $a_{m, n}(x), x \in R$ :

$$
\begin{aligned}
\frac{d \mathbf{A}^{T}}{d x} & =\left(\frac{d \mathbf{A}}{d x}\right)^{T} \\
\frac{d \mathbf{A B}}{d x} & =\left(\frac{d \mathbf{A}}{d x}\right) \mathbf{B}+\mathbf{A}\left(\frac{d \mathbf{B}}{d x}\right) \\
\frac{d \mathbf{A}^{-1}}{d x} & =-\mathbf{A}^{-1}\left(\frac{d \mathbf{A}}{d x}\right) \mathbf{A}^{-1}
\end{aligned}
$$

The term $\frac{\partial \mathbf{M}_{i+1}^{t}}{\partial \alpha_{n}}$ can be obtained using Equation (2):

$$
\frac{\partial \mathbf{M}_{i+1}^{t}}{\partial \alpha_{n}}=\frac{\partial\left(\mathbf{E}_{i+1} \mathbf{M}_{i+1}^{b} \mathbf{E}_{i+1}\right)}{\partial \alpha_{n}}=\frac{\partial \mathbf{E}_{i+1}}{\partial \alpha_{n}} \mathbf{M}_{i+1}^{b} \mathbf{E}_{i+1}+\mathbf{E}_{i+1} \frac{\partial \mathbf{M}_{i+1}^{b}}{\partial \alpha_{n}} \mathbf{E}_{i+1}+\mathbf{E}_{i+1} \mathbf{M}_{i+1}^{b} \frac{\partial \mathbf{E}_{i+1}}{\partial \alpha_{n}} .
$$

The term $\frac{\partial \mathbf{E}_{i+1}}{\partial \alpha_{n}}$ is nonzero only when $i=n$ and the term $\frac{\partial \mathbf{M}_{i+1}^{b}}{\partial \alpha_{n}}$ can be calculated using Equation (4) again, replacing $i$ for $i+1$. Note that to calculate $\frac{\partial \mathbf{M}_{0}^{b}}{\partial \alpha_{n}}$ we also need to calculate

$$
\frac{\partial \mathbf{M}_{1}^{b}}{\partial \alpha_{n}}, \frac{\partial \mathbf{M}_{2}^{b}}{\partial \alpha_{n}}, \ldots, \frac{\partial \mathbf{M}_{n}^{b}}{\partial \alpha_{n}}
$$


It is not necessary to calculate $\frac{\partial \mathbf{M}_{k}^{b}}{\partial \alpha_{n}}$ for $k>n$, because the reflectivity matrix $\mathbf{M}_{k}^{b}$ is affected only by the $k t h$ layer and layers below it. Note that the calculation of the reflectivity as well as its partial derivatives with respect to the elastic parameters should be performed recursively, starting in the lower half-space until it reaches the first interface.

Another point that deserves attention is the computation of the derivatives of the reflection/transmission coefficients of the interfaces, for example, $\frac{\partial \mathbf{R}_{n+1}^{d}}{\partial \alpha_{n}}$. These coefficients are determined by the Knott/Zoepprittz equations and can be put as follows (Aki \& Richards, 2002):

$$
\mathbf{A} x=b .
$$

Where the coefficient matrix $\mathbf{A}$ and the vector $b$ of independent terms depend on the elastic parameters, density and angle of incidence. Differentiating both members with respect to $\alpha_{n}$, for example, and using (4), we obtain:

$$
\frac{\partial \mathbf{A}}{\partial \alpha_{n}} x+\mathbf{A} \frac{\partial x}{\partial \alpha_{n}}=\frac{\partial b}{\partial \alpha_{n}} .
$$

Note that $\frac{\partial \mathbf{A}}{\partial \alpha_{n}}$ and $\frac{\partial b}{\partial \alpha_{n}}$ can be easily obtained from $\mathbf{A}$ and $b$. Once (5) is solved, we just use (6) for the computation of $\frac{\partial x}{\partial \alpha_{n}}$ :

$$
\mathbf{A} \frac{\partial x}{\partial \alpha_{n}}=\frac{\partial b}{\partial \alpha_{n}}-\frac{\partial \mathbf{A}}{\partial \alpha_{n}} x
$$

This linear system has the same coefficient matrix as (5), differing only with respect to the vector of independent terms. As an example, to obtain the term $\frac{\partial x}{\partial \alpha_{1}}$ using the Knott equations we have:

$$
\begin{aligned}
& \mathbf{A}=\left[\begin{array}{cccc}
-p & \frac{\cos i_{0}}{\alpha_{0}} & \frac{2 \rho_{0} \beta_{0}^{2} p \cos i_{0}}{\alpha_{0}} & -\rho_{0}\left(1-2 \beta_{0}^{2} p^{2}\right) \\
-\frac{\cos j_{0}}{\alpha_{0}} & -p & \rho_{0}\left(1-2 \beta_{0}^{2} p^{2}\right) & \frac{2 \rho_{0} \beta_{0}^{2} p \cos j_{0}}{\alpha_{0}} \\
p & \frac{\cos i_{1}}{\alpha_{1}} & \frac{2 \rho_{1} \beta_{1}^{2} p \cos i_{1}}{\alpha_{1}} & \rho_{1}\left(1-2 \beta_{1}^{2} p^{2}\right) \\
-\frac{\cos j_{1}}{\alpha_{1}} & p & -\rho_{1}\left(1-2 \beta_{1}^{2} p^{2}\right) & \frac{2 \rho_{1} \beta_{1}^{2} p \cos j_{1}}{\alpha_{1}}
\end{array}\right] \\
& x=\left[\begin{array}{c}
R_{P P_{1}}^{d} \\
R_{P S_{1}}^{d} \\
T_{P P_{1}}^{d} \\
T_{P S_{1}}^{d}
\end{array}\right] \\
& b=\left[\begin{array}{c}
p \\
-\frac{\cos j_{0}}{\alpha_{0}} \\
-p \\
-\frac{\cos j_{1}}{\alpha_{1}}
\end{array}\right] .
\end{aligned}
$$

The parameters $\alpha_{0}, \beta_{0}, \rho_{0}, \alpha_{1}, \beta_{1}, \rho_{1}$ represent the compressional velocities, longitudinal velocities and densities of the upper and lower mediums, respectively. $p$ is the ray parameter, $\cos i_{0}, \cos i_{1}, \cos j_{0}, \cos j_{1}$ are the cosines of the angles of incidence of compressional waves $(i)$ and shear waves $(j)$; the subscript indicates the medium in which this angle is measured.

Deriving (8) and (9) we have:

$$
\frac{\partial \mathbf{A}}{\partial \alpha_{1}}=\left[\begin{array}{cccc}
0 & 0 & 0 & 0 \\
0 & 0 & 0 & 0 \\
0 & \frac{-\alpha_{1}^{2}-\cos i_{1}^{2}}{\alpha_{1}^{2} \cos i_{1}} & -\frac{2 \rho_{1} \beta_{1}^{2} p\left(\alpha_{1}^{2}+\cos i_{1}^{2}\right)}{\alpha_{1}^{2} \cos i_{1}} & 0 \\
0 & 0 & 0 & 0
\end{array}\right]
$$




$$
\frac{\partial b}{\partial \alpha_{1}}=\left[\begin{array}{l}
0 \\
0 \\
0 \\
0
\end{array}\right]
$$

Thus, substituting in (7) we have:

$$
\mathbf{A} \frac{\partial x}{\partial \alpha_{1}}=\left[\begin{array}{c}
0 \\
0 \\
\frac{R_{P S_{1}}^{d}\left(\alpha_{1}^{2}+\cos i_{1}^{2}\right)+T_{P P_{1}}^{d}\left(2 \rho_{1} \beta_{1}^{2} p\left(\alpha_{1}^{2}+\cos i_{1}^{2}\right)\right)}{\alpha_{1}^{2} \cos i_{1}} \\
0
\end{array}\right] .
$$

Note that you need very little extra effort to solve the later system, since the $\mathbf{A}$ matrix has already suffered $L U$ decomposition for solving (5). For details about the method see Hounie (2011). To implement the inversion method that does not take into account local conversions it is necessary to obtain the Jacobian matrix deriving Equation (3) with respect to the elastic parameters, which was done using the methodology proposed in Oliveira et al. (2009), where the chain rule is used to derive Equation (3):

$$
\frac{\partial r_{p p}^{0}}{\partial \alpha_{n}}=\frac{\partial r_{p p}^{0}}{\partial r_{p p}^{1}} \frac{\partial r_{p p}^{1}}{\partial r_{p p}^{2}} \cdots \frac{\partial r_{p p}^{n-2}}{\partial r_{p p}^{n-1}} \frac{\partial r_{p p}^{n-1}}{\partial \alpha_{n}}
$$

Thus we arrive at:

$$
\frac{\partial r_{p p}^{n-1}}{\partial r_{n}^{p p}}=\frac{\left[T_{n-1}^{d} T_{n-1}^{u}\right] Z_{n}^{-2}}{\left[1-r_{p p}^{n} R_{n-1}^{u}\right]^{2}}
$$

Deriving the reflectivity $r_{p p}^{n-1}$ with respect to $\alpha_{n}$ we obtain:

$$
\frac{\partial r_{p p}^{n-1}}{\partial \alpha_{n}}=\left[\frac{\left(\frac{\partial R_{n-1}^{d}}{\partial \alpha_{n}}+r_{p p}^{n} \frac{\partial b_{n-1}}{\partial \alpha_{n}}+b_{n-1} \frac{\partial r_{p p}^{n}}{\partial \alpha_{n}}\right)\left(1-R_{n-1}^{u} r_{p p}^{n}\right)+\left(\frac{\partial R_{n-1}^{u}}{\partial \alpha_{n}} r_{p p}^{n}+\frac{\partial r_{p p}^{n}}{\partial \alpha_{n}} R_{n-1}^{u}\right)+\left(R_{n-1}^{d}+r_{p p}^{n} b_{n}\right)}{\left(1-R_{n-1}^{u} r_{p p}^{n}\right)^{2}}\right] Z_{n-1}^{2} .
$$

where $b_{n}=T_{n}^{u} T_{n}^{d}+R_{n}^{u} R_{n}^{d}$. Finally, deriving $r_{p p}^{n}$ with respect to $\alpha_{n}$ we obtain:

$$
\frac{\partial r_{p p}^{n}}{\partial \alpha_{n}}=\left[\frac{\left(\frac{\partial R_{n}^{d}}{\partial \alpha_{n}}+r_{p p}^{n+1} \frac{\partial b_{n}}{\partial \alpha_{n}}\right)\left(1-R_{n}^{u} r_{p p}^{n+1}\right)+\frac{\partial R_{n}^{u}}{\partial \alpha_{n}} r_{p p}^{n+1}\left(R_{n}^{d}+r_{p p}^{n+1} b_{n}\right)}{\left(1-R_{n-1}^{u} r_{p p}^{n}\right)^{2}}\right] Z_{n}^{2}-2 r_{p p}^{n} \frac{\partial Z_{n}}{\partial \alpha_{n}}
$$

Note that the reflectivity $r_{p p}^{n+1}$ is not affected by $\alpha_{n}$, then $\frac{\partial r_{p p}^{n+1}}{\partial \alpha_{n}}=0$. These formulas, although extensive, allow to build an efficient iterative algorithm where the reflectivity calculation is performed simultaneously to their derivatives with respect to the elastic parameters of the medium.

\section{REFERENCES}

AKI K \& RICHARDS PG. 2002. Quantitative Seismology. University Science Books. 2 ed. 726 pp.

GOUVEIA WPJ. 1996. Bayesian seismic waveform data inversion: Parameter estimation and uncertainty analysis. Ph.D. thesis, CWP-233, Center for Wave Phenomena, Colorado School of Mines. 153 pp.
HOUNIE JN. 2011. Desenvolvimento de um método multifrequência para a inversão elástica de forma de onda. Doctorate thesis, LENEP, Universidade Estadual do Norte Fluminense. 108 pp.

MALLICK S. 2007. Amplitude-variation-with-offset, elastic-impedence, and wave-equation synthetics - a modeling study. Geophysics, 72 : c1-C7. 
MÜLLER G. 1985. Reflectivity method: a tutorial. J. Geophys., 58: 153-174.

OLIVEIRA SAM. 2009. Uma implementação do método da refletividade para modelagem eficiente de dados sísmicos marítimos e de OBC. Brazilian Journal of Geophysics, 27(3): 323-332.

OLIVEIRA S, LOURES L, MORAES F \& THEODORO C. 2009. Non- linear impedance inversion for attenuating media. Geophysics, 74: R111-R117.

SIMMONS JL \& BACKUS MM. 1994. AVO modeling and the locally converted shear wave. Geophysics, 59: 1237-1248.

ZHAO H, URSIN BR \& AMUNDSEN L. 1994. Frequency-wavenumber elastic inversion of marine seismic data. Geophysics, 59: 1868-1881.

Recebido em 30 setembro, 2012 / Aceito em 24 janeiro, 2014

Received on September 30, 2012 / Accepted on January 24, 2014

\section{NOTES ABOUT THE AUTHORS}

Jorge Nicolás Hounie received a B.S in Geology at the Universidade Federal de Pernambuco in 1997, and a Ph.D. in Petroleum Exploration and Reservoir Engineering at the Petroleum Exploration and Production Laboratory, Universidade Estadual do Norte Fluminense, Brazil. Currently, is a Geophysicist at Petrobras. Research interests include seismic processing and inversion.

Sérgio Adriano Moura Oliveira received a B.S. in Electrical Engineering at the Universidade Federal da Bahia (UFBA) in 1993, and a Ph.D. in Applied Geophysics from the Applied Geophysics Program of the Universidade Federal da Bahia (PPPG/UFBA) in 1998. Currently, is an Associate Professor at the Petroleum Exploration and Production Laboratory, Universidade Estadual do Norte Fluminense, Brazil. Member of the Brazilian Geophysical Society (SBGf) and Society of Exploration Geophysicists (SEG). Research interests include the seismic method in general, seismic modeling, seismic inversion and signal processing. 